\title{
Form perception and attention in pigeons
}

\author{
DONALD S. BLOUGH and PATRICIA M. BLOUGH \\ Brown University, Providence, Rhode Island
}

\begin{abstract}
This report selectively reviews the authors' research on stimulus control by visual forms. Most studies employed visual search of computer-generated displays, and the main behavioral measure was search reaction time. Pigeons classify simple line forms much as do humans, and there was some evidence for feature extraction, though a search asymmetry probe failed to identify several putative features. Dimensional analysis revealed a new quantitative relation between similarity and the probability of detecting a target. It also identified candidates for integral and separable stimulus dimensions. Similarity and set-size variables influenced the extent to which attention was engaged. Further studies addressed the manner in which attention is focused, revealing relationships between priming and search images. In these cases, attention to a selected form is probably driven by a top-down assessment of predictability.
\end{abstract}

Birds seem to interact with their visual world much as do humans, and it is easy to assume that they see things as we do. Psychophysical studies of basic visual functions provide some support for this view, though such things as avian acuity, temporal resolution, and color vision are also unique in suggestive ways (Donovan, 1978; Granda \& Maxwell, 1979). More complex aspects of birds' visual perception have been less well understood, but recent studies of such matters as visual categorization, texture discrimination, and visual illusions are beginning to scratch the surface of our ignorance (cf. Stebbins \& Berkley, 1990; Wasserman, 1993; Zentall, 1993). It is not our purpose to review this literature but rather to summarize some of our own contributions to it. The present paper concentrates on processes revealed by visual search for targets defined by shape or form. Naturally, the extensive literature on human form perception influenced the course of this research, but it also has roots in other areas, such as foraging, and has developed its own logic over time. Our goal has been the general understanding of relevant perceptual processes; pigeon-human comparison is considered where it serves that purpose.

The bird's natural reliance on visual information recommends it for perceptual research. About 20 years ago, we began to harness the pigeon's skillful foraging to a visual search paradigm in which displays and search reaction times (RTs) were computer controlled and recorded (D. S. Blough, 1979). In the research reviewed here, we used this

The preparation of this report was supported in part by National Science Foundation Grant BNS-9407269. The authors thank many long-term members of the psychology department staff at Brown University for their help over the 18-year course of this research. Correspondence should be addressed to either author at Department of Psychology, Campus Box 1853, Brown University, Providence, RI 02912 (e-mail: donald_blough@brown.edu, or patricia_blough@brown.edu).

Note: This article is one of those occasionally invited by the editor in which authors have the opportunity to provide an overview of their research programs.-Editor method to address the interwoven problems of stimulus similarity, perceptual analysis, and attention. Similarity, a truly basic concern of psychological research (e.g., Shepard, 1987), can be approached in many ways; the research we review includes aspects of similarity measurement in the context of dimensional and feature analysis. The idea of features leads to a consideration of perceptual analysis. Much evidence attests that humans parse perceptual objects into parts and dimensions (e.g., Treisman, 1986) and that similarity is affected by this analysis (e.g., Garner, 1978); we review evidence for perceptual analysis in pigeons. Similarity also plays a major role in controlling the disposition of attentional resources, an influence that becomes particularly salient in the visual search task. We review relevant results and go on to discuss a variety of findings related to attentional priming and animal foraging.

This review begins with a brief introduction to the RT measure and to the visual search method. Another section considers findings related to the similarity and perceptual analysis of forms. Finally, we consider the influence of similarity on attention and present an analysis of attentional priming in the search task.

\section{METHODS}

\section{Reaction Time}

The RT measure has been widely used in the study of human perceptual and cognitive processes. Its use depends on the idea that these processes take periods of time that are reflected in RT. As experimenters have determined how long it takes humans to search a display, to recognize a target, to make a decision, or to switch attention, they have been able to narrow the range of viable hypotheses about the mental activities involved. There is good reason to believe that nonhuman subjects can produce equally informative results (D. S. Blough \& P. M. Blough, 1990).

RT has been especially informative in studies of visual search, where it varies systematically with factors such as stimulus similarity or attentional load. RT has the no- 
table advantage over accuracy and response rate in that a single response supplies a meaningful quantity. One consequence is that RTs from many trials yield frequency distributions whose shape may yield information about component processes (Luce, 1986). We found such a case in older research (D. S. Blough, 1978), and we review more recent instances below.

\section{Visual Search}

Visual search lends itself to the study of various problems related to attention and perception. When human or animal subjects look for an object (the "target") in a complex environment, visual representation and attention are implicated, if only because the subjects must know what they are looking for. These are sometimes called top-down processes, because they guide behavior across various stimulus situations. Top-down influences may reflect the influence of such variables as practice in a search task, the number of items in the set of possible targets, target repetition across search trials, and prior cues to target form or location. Also important are stimulus-dependent bottom-up processes, based on stimulus variables such as color, form, and placement of targets and distractors, the number of distractors, and the similarity of target to distractors. Manipulations of all these variables may affect attentional load and thus the accuracy of search and the speed with which a search target is found, or search $R T$.

In the search studies reviewed below, the pigeon subject faced a computer monitor that displayed a number of small black forms on a white background. One form in each display was the target. The target varied in location, and pecks to it brought food reinforcement on an intermittent schedule. An infrared detector system (e.g., D. S. Blough, 1986) enabled the computer to determine the location of the response peck and the time between display onset and the peck, which defined the search RT. Two versions of this task defined the target in somewhat different ways. In fixed-item search, the target was the same on every trial, or it was drawn from a constant, limited set of forms. In odd-item search, the display on a given trial consisted of a number of identical forms, which were distractors, and a single unique form, which was the target. Target and distractors each came from the same set of forms; each form in the set was equally often a target and a distractor, and all possible pairs of forms in the set appeared equally often in an experimental session.

Birds typically perform these search tasks with high accuracy and require infrequent reinforcement, so sessions provide 1,000 or more correct-detection RTs. Because the task is adapted to the pigeon's natural behavior, it has certain features that distinguish it from typical studies of human search. Among these are the following. (1) Often, the human searcher's task is to indicate the presence or absence of a target in a display, and he or she may be unable to report its location (Treisman \& Gelade, 1980). In contrast, the pigeon's RT reflects the time required both to detect and to locate the target. (2) When human search is limited to detection, it is subject to response bias, whereas the pigeon's task is a forced-choice procedure relatively free of bias. (3) Some human tasks involve absolute identification, with the searcher indicating which of several possible targets appeared on a trial; the pigeon's response does not categorize the target form. (4) Many human studies use very brief displays, whereas the pigeon search response terminates a trial, and display time thus covaries with RT. This difference has prompted steps in the pigeon studies to minimize systematic effects of motor components such as head and eye movements. For example, display-size studies control the area occupied by the search displays, so that motion times average the same across conditions. Finally, the reader should be aware that issues addressed in the following pages are not coextensive with the use of visual search; where appropriate, we refer to research based on other methods.

\section{SIMILARITY AND PERCEPTUAL ANALYSIS}

\section{Classifying Forms to Describe Their Appearance}

What does an object look like to another person or to a bird? The most direct answer to this question is given by direct or implied comparisons with other objects. "Blood is red" means that blood elicits the same color response as certain other things; to most people, it looks like ketchup and not like pea soup. Blood was also "red" for the famous chemist John Dalton, but for him this implied unusual classifications: "Blood appears to me not unlike that color called bottle green" and "I have seen specimens of crimson, claret, and mud which were nearly alike" (Boring, 1942). The comparison of Dalton's classifications with those of other observers had deep implications for the understanding of color perception. Comparisons among the classifications of different species may contribute in like manner to an understanding of form perception.

One does not expect comments like Dalton's from a pigeon, but pigeons may supply classifications that open a similar window on their perceptual world. Several lines of research (e.g., Herrnstein, 1979; Wasserman, KirkpatrickSteger, Van Hamme, \& Biederman, 1993) have studied discriminations that permit classifications of some complex real-world objects. Taking a different tack, our initial research provided for comparisons among many forms that lent themselves to objective description and could provide a pattern of interlocking categories. These forms were simple and small, so the visual search task was an efficient way to make the many cross-comparisons needed to develop patterns of classification.

We first adopted the search strategy to explore the pigeon's perception of the letters of the alphabet (D. S. Blough, 1982, 1985). While unnatural, letters have some merit for this purpose. They share line segments and configurations to varying degrees, and they constitute a large enough set that substantial patterns of classification may appear. With such simple stimuli, it may be relatively easy to develop relations between perceptual categories and physical characteristics. In addition, several experiments provide comparative data on human letter classifications. 
The first experiment (D. S. Blough, 1982) used a simple version of fixed-item search in which each letter of the alphabet was used as the search target for four sessions. This target letter (e.g., "N") appeared on a monitor screen on every trial, together with two copies of a different letter; the nontarget letter was semirandomly chosen on each trial such that all such letters appeared equally often during a session. The position of the three letters varied randomly over three locations. After four sessions $(2,700$ trials), a new target letter was introduced, and so on. The percent of pecks to the target on its last two sessions was calculated for each pairing of that target with each other letter. Thus, each letter pair yielded two accuracy measures (e.g., one from the combination target $N$-distractor $A$ and the other from the combination target $A$-distractor $N$ ). The results were summarized in a $26 \times 26$ cell table. (The main diagonal, describing the discrimination of a letter from itself, was empty, although the similarity of an object to itself is a matter of some interest; see, e.g., Tversky, 1977). Accuracy in this first letter study varied from chance with letter pairs such as $A$ and $R$ to above $80 \%$ for letters such as A and I.

A related study (D. S. Blough \& Franklin, 1985) used the letters of the alphabet in a variant of the odd-item search method described above. A matrix of letters equally spaced in 9 rows and 17 columns appeared on the screen. The pigeon searched for a small square cluster or patch of 9 identical letters on a larger background composed of many copies of one of the other letters (e.g., a group of $9 \mathrm{Ns}$ on a background of 144 As). As specified by the odd-item procedure, the target and the background letters both changed from trial to trial, and all letters and letter pairs appeared equally often. Again, a $26 \times 26$ matrix summarized the results. Though the stimulus displays in these two studies were quite different, the results were similar, with accuracy scores correlating .89.

The search accuracies in these experiments provide the data needed to classify the letters by their similarity to the pigeon subjects. Hierarchical cluster analysis gave an effective display of these results. Figure 1 shows the results of the two studies, reduced in this way. The computer algorithm (CLUSTER; SAS, 1982) grouped the letters together according to interletter distances in a 26-dimensional space. Letters that were closest together in this space were grouped first and appear toward the bottom of each diagram. A cluster's height in each diagram is proportional to the logarithm of the greatest distance between any pair of letters in the cluster at that level.

The clusters in Figure 1 are classifications showing to some degree what letters look like to pigeons. Human observers find these pigeon classifications similar to their own, and the clusters correspond reasonably well to those based on human letter judgments (D. S. Blough, 1985; Podgorny \& Garner, 1979). The results thus suggest that the perceptual processing of these very simple forms is similar in pigeons and humans despite the wide evolutionary gap that separates these species. Though it would have been interesting to unearth a pigeon "Daltonism" show-
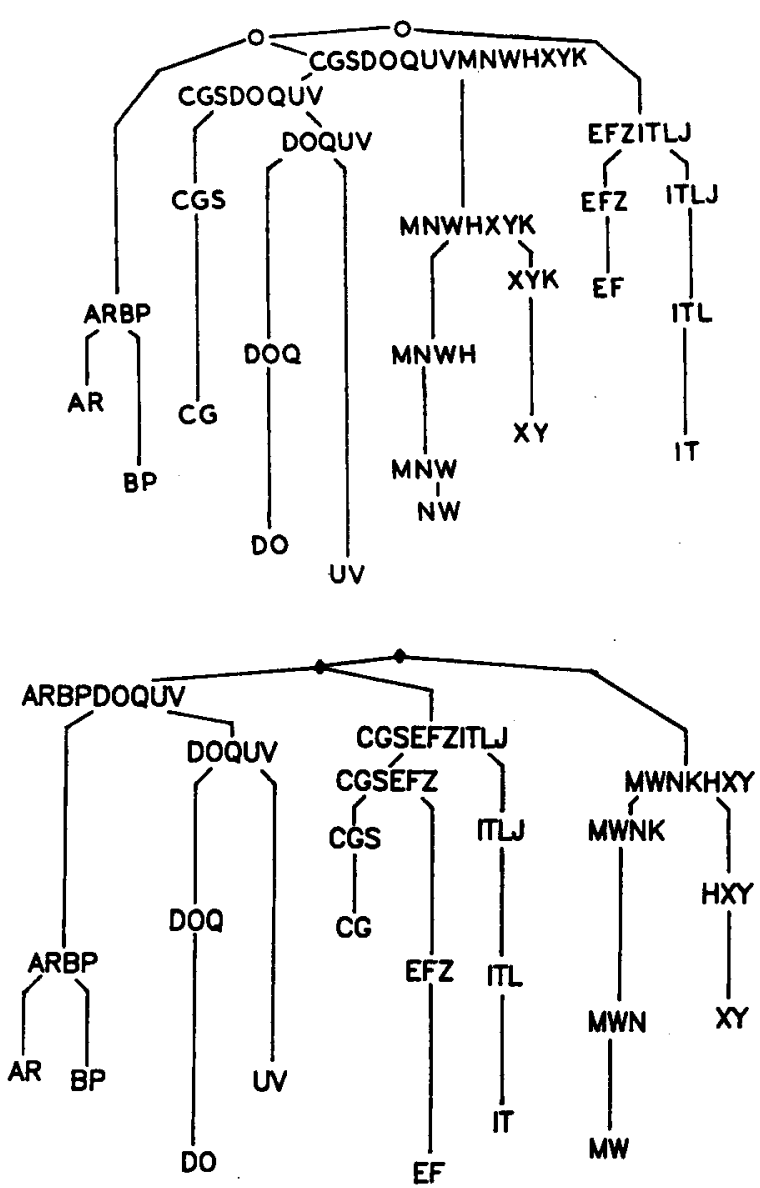

Figure 1. Hierarchical cluster diagrams from each of two studies with pigeons. Top panel: Based on data from a consistent search procedure with a display size of three (D. S. Blough, 1982, 1985). Bottom panel: Based on data from an odd-item texture search task (D. S. Blough \& Franklin, 1985). Despite substantial differences in task and display parameters, the clusters are similar in many respects and correspond well to human similarity ratings.

ing cross-species differences in form perception, the degree of similarity between pigeon and human results is perhaps equally remarkable.

\section{Theories of Similarity}

It developed in the preceding discussion that stimulus classifications can be derived from many measurements of interstimulus similarity. Similarity is a truly fundamental psychological concept (e.g., Shepard, 1980), and it has received considerable theoretical analysis. Three conceptualizations of similarity dominate the literature; they may be termed models to the extent that they help to explain and predict observed measurements. The least analytic and perhaps most obvious approach is the template model. The simplest such model measures form similarity as the degree to which two-dimensional representations of stimuli overlap when one is placed on top of 
the other. Serious difficulties attend this approach (e.g., even small magnification or rotation of one form may drastically affect its overlap with another), and it was only moderately successful in predicting the letter data presented above (see D. S. Blough, 1985). However, sophisticated versions of the model have shown promise; for example, a variant of the template model has handled some pigeon form perception data remarkably well (Heinemann \& Chase, 1989).

Two other approaches to similarity assume the perceptual analysis of stimuli into components. This assumption is justified by extensive psychophysical and physiological evidence indicating that different aspects of visual stimuli are processed somewhat independently (for a review and analysis, see Treisman, 1986). The feature approach considers an object as a collection of attributes; shared features increase the similarity of two objects, whereas unshared features decrease similarity (e.g., D. S. Blough, 1991; Tversky, 1977). Of course, this approach requires the definition of features, a problem to which we return below. The dimensional approach describes objects in terms of continuous dimensions such as brightness, color, and size. This approach facilitates the representation of similarities as distances between the objects in a space of low dimensionality (e.g., Shepard, 1980). As we shall see, such a spatial representation is very convenient for some purposes.

These conceptualizations of similarity are useful, but they are not complete theories of form perception (they say little, for example, about context effects; e.g., Fujita, D. S. Blough, \& P. M. Blough, 1991) and they are not necessarily incompatible with each other. Though significant theoretical issues surround the choice of one or another (see, e.g., Garner, 1974; Prinzmetal, 1995; Shepard, 1980; Treisman, 1986; Treisman \& Gormican, 1988; Tversky, 1977; Tversky \& Gati, 1982), different kinds of stimuli lend themselves better to one treatment than to another, and most experimenters choose stimulus sets with this in mind. An interest in dimensions and their combination might suggest the use of forms varying in size, whereas an interest in features might suggest the use of forms composed from a list of discrete parts.

\section{Features of Simple Forms}

The features composing a form may be defined in various ways. At one extreme are the simple primitives suggested by Julesz (1981) and others (e.g., "line end," "crossing"). At another level are parts, such as cylinders or cubes, that might compose a complex three-dimensional object (e.g., Biederman, 1987). The latter approach has received an interesting application to pigeon perception by Wasserman and his associates (e.g., Wasserman et al., 1993). In the interests of simplicity, and to favor quantitative analysis, we have chosen to use mainly simple forms whose parts may correspond to line segments, loops, corners, and the like.

Features suggested by form classification. We have seen that the study of perceptual appearance can begin by sorting objects into similarity groups. Once this is done, it may be possible to identify potential components that are shared by members of the same group but not by members of different groups. For example, the reader may confirm in Figure 1 that the pigeon's groupings of alphabetic characters suggest features such as "open center" (DOQ), "loop at top" (ABPR), and "vertical stroke" (ITL). However, the discovery of putative features corresponding to groups does not demonstrate that extraction of those features plays a role in the perception of the forms. Alternative hypotheses may do as well or better; for example, as noted above, a template model handled the letter data moderately well (D. S. Blough, 1985).

Despite their limitations, carefully designed classification experiments can be used to test alternative processing hypotheses. For example, each of the three forms shown in Figure 2 overlaps the others to an equal degree when they are superimposed, so the simplest template model would make no differential predictions about them. Also, the three forms are composed of the same line segments, so they are equated for those simple potential features. If features are defined in terms of line segments, corners, and line endings, with relative position irrelevant, two of the forms ( 1 and 2) have the same features, but the other pairs differ in this respect. If these were key functional features, as Julesz (1981) and others have argued, the similarities among the forms as measured in the search task should vary correspondingly. If features are also defined in relational terms, such as "right-facing gaps" and "connectedness," as some of the letter data suggested, a different set of similarities might emerge.

We tested the forms shown in Figure 2 in two odd-item search tasks run the same way as the letter experiments described above. Different orientations and rotations of the three forms were used, together with some other related forms (D. S. Blough \& Franklin, 1985). The results showed that searches involving the "S" (Form 2) and the "E" (Form 3) together, one as target and the other as distractor, were more difficult than any search involving the disconnected "rectangle+line" (Form 1). These results ruled out a simple template account, because the forms overlapped equally. They also argued against a dominant role for specific line endings and junctions (Julesz, 1981), because Forms 1 and 2 are identical in this regard (same corners, same number and orientation of line endings). "Connectedness" seemed to be the most important factor controlling similarity for the pigeons, but search tasks with humans have shown this difference to be of low

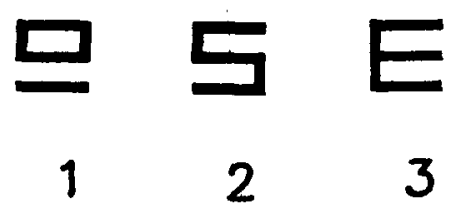

Figure 2. Three forms used in exploratory tests of feature definition (D. S. Blough \& Franklin, 1985). Forms 1 and 2 have the same local features (line segments, line endings, and corners) but differ in connectivity. Forms 2 and 3 are both connected but differ in some local features. All three forms overlap equally when superimposed. 
salience (e.g., Julesz, 1981). In a related study (Todrin \& D. S. Blough, 1983), pigeons discriminated rotations and reflections of $U$-shaped forms and of $F$-shaped forms. The birds more easily confused mirror-image pairs than $90^{\circ}$ rotated pairs, and they confused left-right mirror reflections more than up-down mirror reflections. These results again seem incompatible with a template account, but, in this case, they support the general comparability of form classification in pigeons and humans. Taken together with classification data collected by other methods (e.g., Delius \& Hollard, 1995), these studies suggest that an intriguing pattern of cross-species relationships between pigeon and human form perception remains to be explored. We return to other aspects of this matter below.

Identifying features through search asymmetry. We have seen that classification by similarity may suggest features but does not demonstrate that the perceptual system uses them. A more functional definition is provided by the phenomenon of search asymmetry, given some assumptions about the underlying processing. In a search asymmetry experiment, subjects look for a single target concealed somewhere in a field of identical distractors (e.g., a Q surrounded by Os). The target differs from the distractors only by the presence of a putative feature in one form and its absence in the other. Thus, the tail of the $\mathrm{Q}$ might be a feature. If the target contains the feature, it tends to "pop out" from the background; search RT is low and does not change much as the number of distractors is increased. If the distractors contain the feature and it is absent from the target ( $a n$ O on a field of Qs), search RT is relatively high and increases with the number of distractors. The differences in search RT obtained under the two conditions define a search asymmetry and identify the variable form part as a feature.

A number of the forms that have been tested for search asymmetry in humans are composed of lines, and it is natural to identify a line segment (such as one side of a rectangle) as a potential feature. If adding a line segment to a form added a feature, one would expect that pairs differing by the segment to show a search asymmetry favoring the form with the segment. This is often the case, as exemplified by $\mathrm{O}$ and $\mathrm{Q}$. However, the features found for human observers are not always, or even typically, additions. For example, search asymmetry defines the gap in $\mathrm{C}$ as a feature, when $\mathrm{C}$ is contrasted with $\mathrm{O}$. Likewise, a slightly curved line is found to contain a feature, when the curve is contrasted with straight lines (Treisman \& Gormican, 1988). (It should be noted that features defined in this way are limited to the testing context and the specific forms that experimenter chooses to try.)

What assumptions lead to the acceptance of search asymmetry as defining a feature? A common idea is that, for an aspect of a form to be a feature, detectors for that aspect must be distributed across the visual field (e.g., Julesz \& Schumer, 1981). Detection is subject to Weber's law (Treisman \& Gormican, 1988). In a display with many elements, when the feature is in the target, the subject can readily detect the relatively large difference between a target-absent (zero-feature) display and a target-present (one-feature) display. However, when the feature is in every item of an $N$-item display except the target, it is difficult to detect the difference between a target-absent ( $N$-feature) display and a target-present $(N-1$ feature) display. Search RT goes up with display size in the latter case because attention must be focused successively on different items or areas in the field. Though this interpretation remains largely hypothetical, search asymmetry provides an objective measure that can be used to test the functional characteristics of form parts or aspects.

Our exploration of search asymmetry in pigeons began with demonstrations that our methods could detect the phenomenon where it was strongly expected. An asymmetry favoring larger stimuli is reported for humans (e.g., Treisman \& Gormican, 1988) and monkeys (Schiller \& Lee, 1991). The results showed search asymmetries favoring forms that covered a relatively large area; for example, search for a large black spot on a background of small spots was faster than the reverse (D. S. Blough, 1987), and search among forms composed of from one to four line segments produced asymmetry favoring targets with more segments (D. S. Blough \& P. M. Blough, 1990, p. 266). These data agree with human results (Treisman \& Gormican, 1988) and established the potential usefulness of the method with pigeons.

We next used search asymmetry to test some simple features with pigeons; this work replicated at the same time previously reported data on human subjects, using a method as comparable as possible to that used with pigeons (Allan \& D. S. Blough, 1989). The studies were done using both consistent search, in which the subjects learned a specific form as target, and odd-item search, which permitted within-session comparison of targetdistractor pairings. The crucial tests involved as putative features a vertical line or a gap. When present, the line crossed the bottom of a circle or the middle of an S-shaped form. When present, the gap appeared in one side of a triangle under some conditions and in a square under other conditions.

The results of the search asymmetry tests showed that both the line and the gap were clearly defined as "features" for our human subjects under both test conditions. This replicated for our conditions' previously well-established results (Treisman \& Gormican, 1988). Surprisingly, given the similarity between other pigeon and human results, the pigeons failed to show the expected asymmetry. In fact, there was a small but significant reverse "gap" effect for the pigeons. This result is illustrated in Figure 3.

These results on search asymmetry provide another suggestion that perceptual processing in pigeons may differ in certain ways from that in humans. Several interesting hypotheses as to the source of this difference remain to be tested. For example, search may be similarly influenced by features in the two species, but the features used may sometimes differ. We noted above the apparent influence of "connectedness" in similarity among forms for pigeons, and the reverse gap effect with pigeons could 


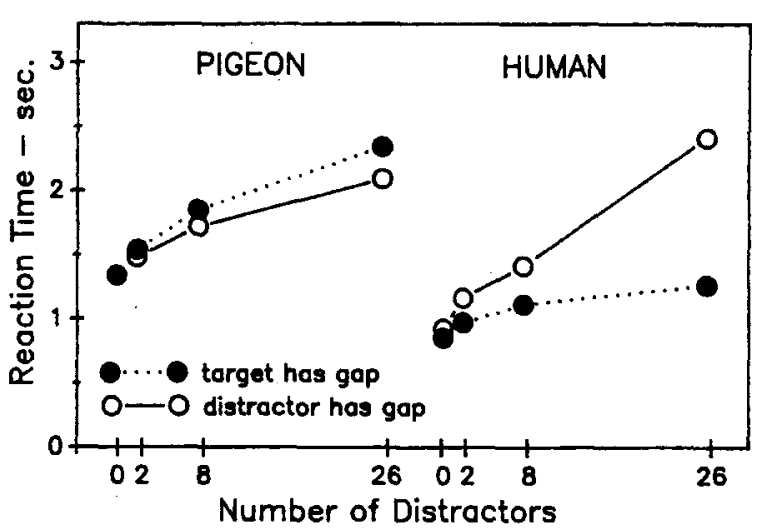

Figure 3. Reaction times from searches among small triangles. The target triangle had a gap in it and the distractors were complete triangles or vice versa. The data of human subjects showed a search asymmetry defining the gap as a feature; the reverse is true for pigeon subjects (Allan \& D. S. Blough, 1989).

implicate this factor in pigeon search asymmetry. Another possibility worth exploring with both species is the effect of training. The pigeons had much more training with the specific forms and the search task than has usually been the case with human subjects. On the other hand, the overtraining humans have had with letters and some other forms might affect the features that human subjects will identify (possibly, for example, the gap defining a C). Using forms that lacked pop-out features, Treisman, Vieira, and Hayes (1992) found that extended training produced dramatic drops in search RT, but transfer tests failed to confirm that features had been induced by the training. Most commentators have assumed that feature detectors are hard-wired into the form processing system (e.g., Treisman \& Gormican, 1988), and there clearly is need for further exploration of this key issue, some of which might efficiently be done with pigeon subjects.

Identifying features through RT covariation. As discussed in later sections, a relatively low search RT for a given form may serve to indicate that the form is being attended to at the time of measurement. In like manner, a low RT during search for several related forms could signal attention to some aspect common to those forms. For example, a pigeon pecking in a pan of mixed grain might sometimes take certain grains more rapidly than others. For a period of time, the chosen grains might turn out to have a dark marking in common. At another time, the chosen grains might be relatively large. The key idea is that searches dependent on the same feature might all speed up when attention is directed to that feature and slow down when attention shifts to another feature. If so, the covariation of RTs across forms could be used to identify a feature common to the forms.

A recent study in our laboratory (D. S. Blough, 1993b) sought RT covariations of the sort just suggested. One experiment used a set of simple box-shaped forms (Figure 4). To the human eye, these forms have common features, such as sides and corners, and two distinguishing features: a gap at the top and/or a short vertical line at the bottom. The question was whether those aspects functioned as separable features for the pigeon. The experiment used odd-item search, pairing as target and distractor those eight combinations in which the target differed from the distractor only with respect to the line or with respect to the gap. As always with this method, each form appeared equally often as target or distractor. Sometimes, the line was in the target; sometimes, it was in the distractor. Sometimes, the gap was in the target; sometimes, it was in the distractor. But, on any trial, the target and distractors could only be distinguished by their difference with respect to the line or with respect to the gap, never by both.

The analysis assumed that the pigeon might attend to different aspects of these forms at different times and that attention might drift between these aspects. For example, if the pigeon was looking for the gap during a series of trials, its search RT would be relatively low when the target contained the gap and the distractors did not. If the pigeon was looking for the line, targets with lines would be found rapidly on backgrounds with no lines. The analysis could yield less obvious features as well, each with its own predicted pattern of RT results: "look for solid top" (absence of gap), "look for unbroken bottom" (absence of line), "attend to the top of the form" (low RT for gap in either target or distractors), and "attend to the bottom of the form" (low RT for line in either target or distractors).

A major outcome of the experiment just described (D. S. Blough, 1993b: Experiment 1D) was that two factors accounted for much of the RT variance across sessions. These are graphically summarized in Figure 4, which was based on multidimensional scaling (to be discussed below) and confirmed by cluster analysis. The

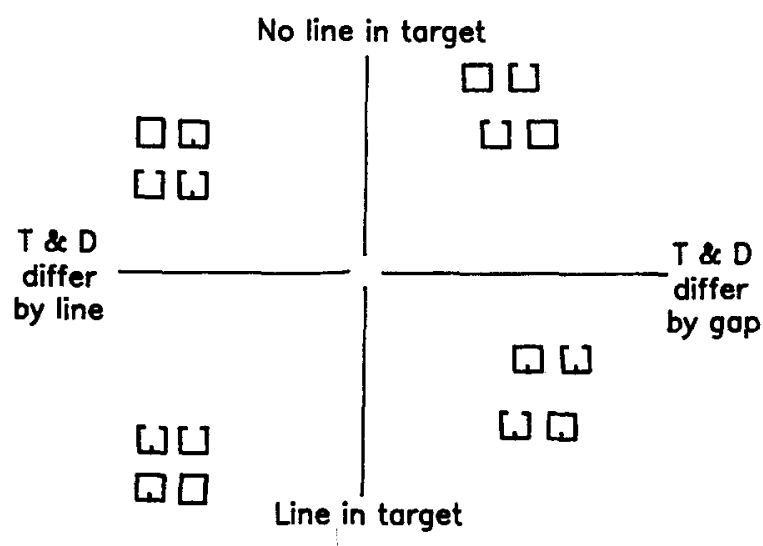

( TARGET IS LEFT ITEM IN EACH PAR )

Figure 4. This result of multidimensional scaling is based on correlations among search types across sessions. The distance between any two target (T)-distractor (D) pairs reflects the covariation of RTs to those pairs; short distances imply large covariation. The vertical dimension may reflect fluctuations in attention to the line sometimes present at the bottom of the form; the horizontal dimension may reflect fluctuations in attention between the top and the bottom of the rectangular form (D. S. Blough, 1993b). 
vertical dimension implicates the line or its absence as a feature; other analyses indicated that 2 of the 3 subjects were in fact looking for the line as a defining feature of the target. The factor implicated by the horizontal dimension is perhaps more interesting and surprising. The simplest way to interpret this is that the birds shifted their attention among different locations within the forms. Thus, the bird sometimes attended relatively more to the top of the form, where the gap might appear, and sometimes attended relatively more to the bottom of the form, where the interior line might appear. This is particularly interesting because the locations in question were only a few millimeters apart and were defined only within forms; the forms themselves were placed randomly. Evidence too extensive to repeat here argued that these two factors, "attention to line" and "attention to location within form," fluctuated independently of each other, suggesting the involvement of independent processes. Also, neither gap nor absence of gap emerged as a feature, though forms that differed by a gap were about as well discriminated as forms that differed by a line. This is especially interesting in light of the unexpected failure of gap to meet the search asymmetry criterion for a feature in pigeons.

In summary, several methods show promise in enabling the identification of features that play a functional role in pigeon form perception. Some suggestive results on the identity of features emerged, though we clearly have not moved far in constructing a feature list. This could be a direction for future research, particularly if it were conjoined with explorations of the genesis of features (e.g., the role of past experience) and the attentional processes that control their independence or interdependence. We will return to attentional matters later, but, next, we review a somewhat different approach to the similarity issue.

\section{The Dimensional Approach to Form Similarity}

As we have seen, stimuli with physically definable parts or discontinuities can be used to help identify psychologically separable features. Such features are usually treated as binary components: all or none, present or absent. Stimuli that differ in an orderly way along continua may provide a different kind of information. For this purpose, experimenters have usually devised simple stimuli that vary along physically definable continua, such as length or angle of tilt. One may then ask whether the responses of subjects generate a psychological similarity space that reflects the stimulus dimensions, and, if so, how the effects of dimensions combine. Geometric models provide a framework within which these questions may be addressed. These models represent stimulus objects as points in a psychological similarity space-usually, a space of from one to four dimensions. The distance between any pair of points in the space represents the dissimilarity of a pair of objects. Computer algorithms for multidimensional scaling (MDS) derive the dimensions and the point locations that best fit a set of input data, which could, for example, be similarity judgments from human subjects or discriminative RTs from animals. Inspection often reveals the psychological nature of the derived dimensions, and the geometry of the space may suggest how subjects integrate dimensional information. (A number of texts discuss varieties of MDS and their applications; see, e.g., DunnRankin, 1983.)

As one would anticipate, the particular scaling algorithm that is chosen will affect to some extent the psychological dimensions discovered and the distribution of points in similarity space. However, with the stimulus sets we have used, we have not found much difference between the outcomes of several somewhat different sets of assumptions. We have usually used Shepard and Kruskal's nonmetric MDS procedure (Kruskal, 1964; Shepard, 1962) as implemented in the ALSCAL computer procedure (SAS, 1982). This procedure assumes little about the input data; in particular, it retains only the ordinal relations among input values. The program distributed the stimulus items in space such that the rank order of distances between pairs of stimuli was as nearly as possible the exact inverse of the rank order of RTs for successful searches involving the pairs. It is a surprising fact that the rank order of such input data suffices to determine quantitative metric distances in the psychological similarity space (Shepard, 1980).

We have used geometric analysis to identify functional aspects of stimuli, the relative weight or salience of such aspects, how aspects may combine, and how similarity and search RT may be quantitatively related to each other. The speed (RT) or accuracy of visual search in the odditem search task has supplied the raw material for these analyses. The pairing of each stimulus with every other stimulus, with each serving as a single target and again repeated as a field of distractors, provided the multiple comparisons required as input for MDS. In the following sections, we review some of this research.

How dimensions combine. As noted above, MDS algorithms locate stimulus items with respect to psychological dimensions. The relation between these psychological dimensions and the physical characteristics or dimensions of the stimulus objects can help to clarify the nature of perceptual processing. This may be illustrated by comparing scaling results based on odd-item search with two sets of forms. Each stimulus set varied in two physical dimensions. Each dimension assumed one of four values, yielding a total set of 16 items. Each item in the first set was a small $U$ located immediately above a black rectangular block; the $\mathrm{U}$ varied in width, and the block varied in height (Figure 5). The items in the second set were outline rectangles varying in height and width. To minimize potential perceptual discontinuity (e.g., "tall" vs. "wide" items), all of the rectangles in the second set were wider than any was high (Figure 5).

The two sets of items were studied in separate experiments (D. S. Blough, 1988). Each item in a set was paired in a search display with every other item, providing a matrix of search RTs that was taken to reflect the pair- 


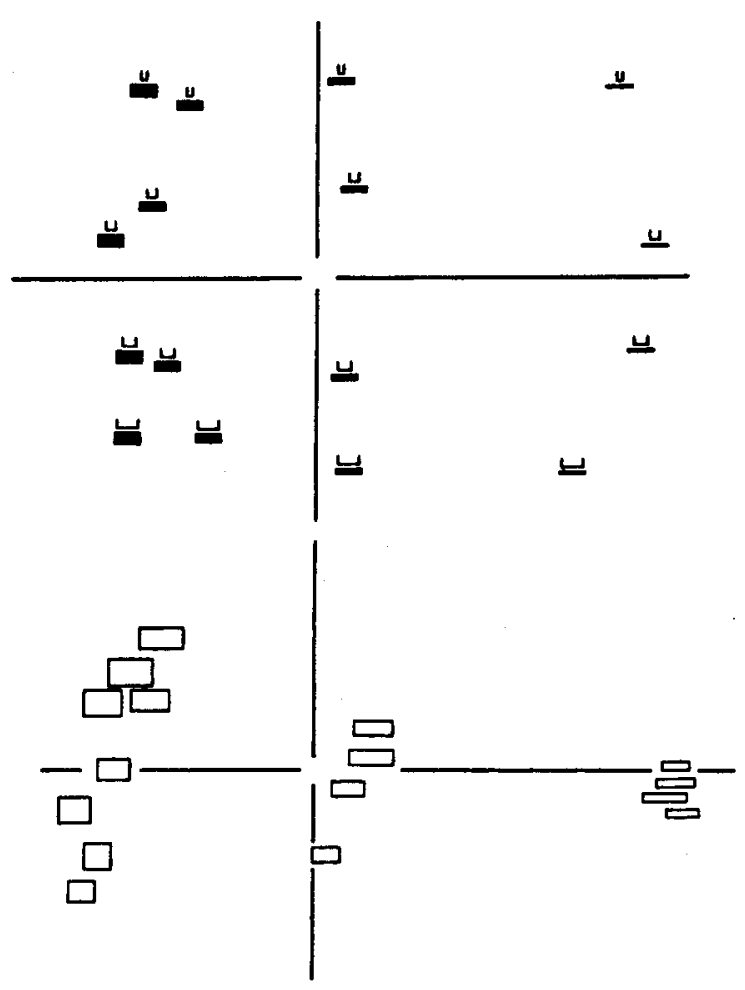

Figure 5. Nonmetric multidimensional scaling results from two sets of forms. Forms within each set were paired in odd-item displays. Similarities among block $+U$ forms (top panel) were well predicted by either a Euclidean or a city-block combination of the two physical dimensions (the Euclidean fit is shown). Similarities among rectangles (bottom panel) showed a strong interaction between height and width, suggesting that these physical dimensions are not mapped into corresponding psychological dimensions (D. S. Blough, 1988).

wise similarities of the items. Nonmetric (ordinal) scaling of the items produced the similarity structures depicted in Figure 5.

The results shown at the top of Figure 5 indicate that the psychological dimensions discovered for the U-block items corresponded to the physical variations of those items. The prominence of the horizontal dimension suggests that the physical change in block size was more salient than the change in U size. Also, the rather symmetrical shape of the display suggests that these dimensions did not interact substantially. This impression of independence was supported by an analysis of RTs, which showed that variations on one dimension had about the same effect regardless of the level at which the other dimension was held constant (D. S. Blough, 1991). In contrast, the configuration for the set of rectangular items (Figure 5, bottom) shows marked interaction between the input dimensions of height and width. Vertical changes dominated, with the horizontal playing an increasing role with larger forms. Human judgments of rectangles show similar interaction (e.g., Burns, Shepp, McDonough, \& Wiener-Ehrlich, 1978).
The differences exemplified in Figure 5 call to mind a major issue in the literature on perceptual dimensions, concerning whether dimensions are analyzed independently ("separable" dimensions) or are processed together as a perceptual unit ("integral" dimensions), or possibly in some other way (see Garner, 1974, 1978). There is some evidence for the hypothesis that these ways of processing correspond to different rules of dimensional combination: Separable dimensions combine additively, by the "city-block" rule, by which a similarity change $a$ on one dimension adds to a change $b$ on the other dimension to yield a similarity change of $(a+b)$. Changes on integral dimensions tend to follow the Euclidean formula for distance in two-dimensional space: $\left(a^{2}+b^{2}\right)^{1 / 2}$. By this test, the data sets just considered (Figure 5) showed a slight indication of greater integrality for the rectangle set than for the U-box set, but the fits were not very different (D. S. Blough, 1988), perhaps because the number of stimuli was limited. A previous generalization paradigm provided a more detailed test of dimensional combinations (D. S. Blough, 1972). Test stimuli differed in two dimensions (wavelength and tone frequency, or wavelength and line tilt) from a single reinforced $\mathrm{S}+$ combination. This paradigm yielded a rather clear combination rule, but this rule did not conform to any ordinary spatial geometry. The rule, which we called multiplicative interaction, was such that the larger stimulus change dominated the smaller to differing degrees, depending on their distance from an $\mathrm{S}+$ reference point. Thus, a city-block metric held for stimuli near the $\mathrm{S}+$ combination but farther away the combination rule became Euclidean, and so on. Our data are as yet inadequate to test this rule in the search paradigm.

A quantitative relationship between similarity and RT. The MDS analyses reviewed above yielded interform similarities, while assuming only a monotonic relation between RT and similarity. It would be quite useful to state a stronger relationship between these variables, if possible, especially for the purpose of quantitative modeling. Extensive data to address this question were collected in a series of odd-item search tasks (D. S. Blough, 1988). The stimulus sets included forms varying in size and/or shape and differing in possible dimensional structure. As usual, all forms within a set were paired as target and distractor in all possible combinations, yielding in each case a matrix of search RTs. These were then subjected to nonmetric MDS as described above, yielding psychological distances between all pairs of stimuli in each set. From these, it was found that, for all data sets, RT declined approximately as an exponential decay function of distance, when a constant $K$ (representing an RT "floor") was subtracted from RT. Expressed in its logarithmic form, this function is

$$
\log (\mathrm{RT}-K)=a * D+b,
$$

where $D$ is psychological distance, and $a$ and $b$ are constants. In other words, $\log (\mathrm{RT}-K)$ is a linear function of similarity, and $\log (\mathrm{RT}-K)$ provides an equal interval scale of similarity. This relationship is exemplified by 


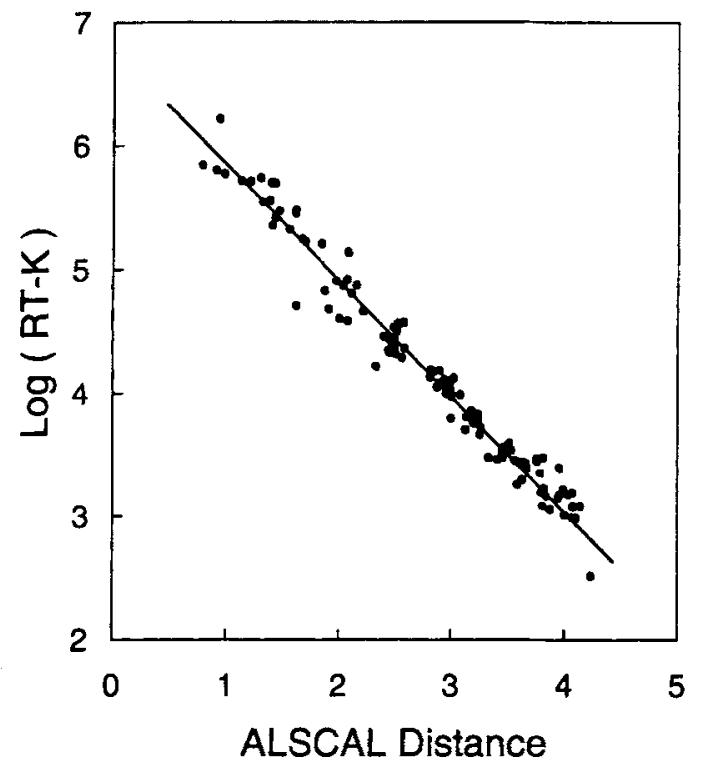

Figure 6. An example of data conforming to the relation expressed in Equation 1 (see text). The distances between each pair of forms in the top diagram of Figure 5 is plotted against $\log (\mathrm{RT}-K)$, where each $R T$ is the mean reaction time for searches involving that pair of forms. (The same constant "floor" $K$ is subtracted from all RTs.)

Figure 6 , which shows $\log (\mathrm{RT}-K)$ plotted against the distances between the points in Figure 5 (top).

The exponential relation just stated links stimulus similarity to the mean search RT for each of many pairs of forms. It is analogous to the exponential relation between distance and the probability of generalization (e.g., Shepard, 1987; see D. S. Blough, 1988, for further discussion). What it may mean is clarified by interpreting it in conjunction with an analysis of RT frequency distributions. This analysis stems from the idea, dating at least from Donders (see Luce, 1986), that RT reflects the sum of times taken by a series of events that occur between stimulus and response. Many data sets suggest that these events are of two kinds, each of which contributes a component to observed RT. Some events vary with the stimulus and related task parameters, such as expectation. For search, these might control the probability that a target would be found at any given moment. Other events are relatively unrelated to perceptual decision making and might include sensory transduction, neural transmission, and motor movement; these events contribute a second component to observed RT. According to this scheme, an observed RT is the sum of the two component intervals, each randomly selected from its own underlying distribution. If this is the case, the distribution of observed RTs corresponds to the convolution of the two underlying distributions.

The reality of serial, additive RT components has not been generally demonstrated, but the scheme just suggested has been remarkably fruitful nonetheless (Luce,
1986). We undertook to look at search RT data from this perspective. An odd-item search experiment provided the raw material (D. S. Blough, 1988, Experiment 2). It yielded a large number of RTs from each of six target-distractor pairs differing in similarity. All six of the resulting RT distributions were well described by the convolution of two distributions - one exponential, the other Gaussian (i.e., normal). (This same convolution has been favored in the analysis of human RTs as well; e.g., Luce, 1986; Ratcliff, 1978.) Data from three target-distractor pairs that differed in similarity appear as points in Figure 7. Theoretical fits, shown by solid curves, are convolutions of an exponential and a Gaussian. Changes in a single pa-
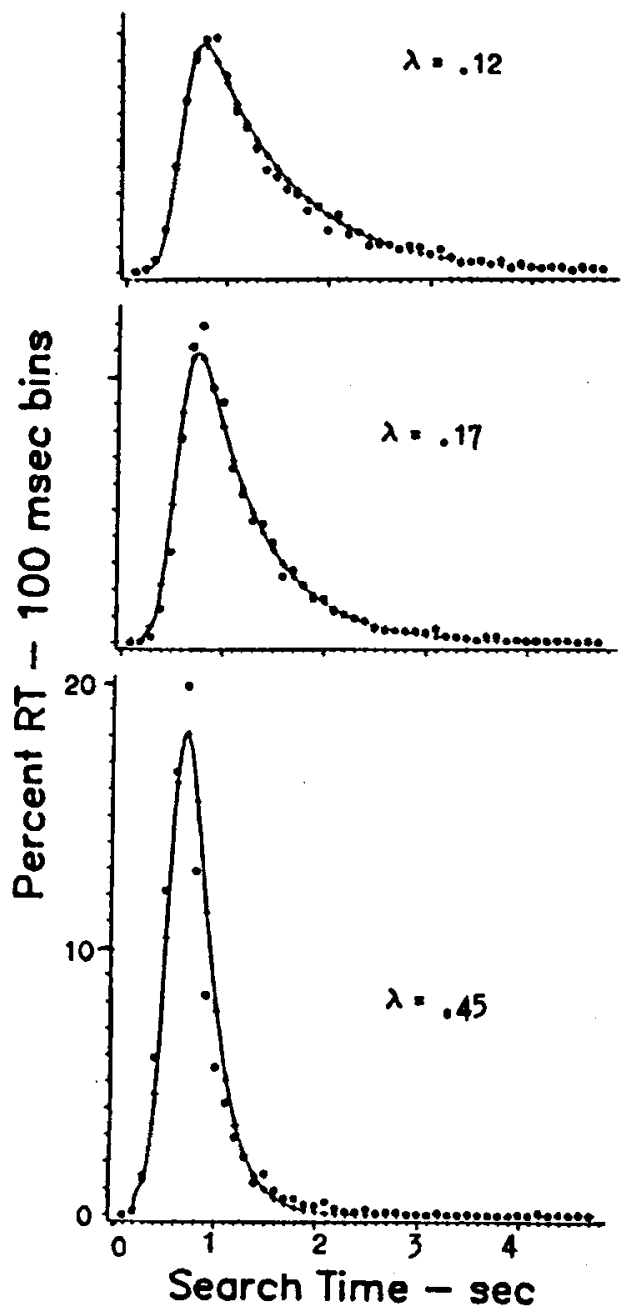

Figure 7. Frequency distributions of RTs for three searches differing in target-distractor similarity; the target was larger than the distractors by varying amounts. The points in each panel represent 2,178 presentations of a single type. Data are combined across birds in a manner that retains the shape of individual curves. The curves represent an approximation to the best fit of the points by a convolution of a Gaussian (the same for all panels) and an exponential decay function, which varied only in the decay parameter given next to each curve (D. S. Blough, 1988). See text. 
rameter sufficed to achieve all fits. This parameter was the decay constant of an exponential distribution, which represented the search or perceptual decision-making component of RT. This component varied with targetdistractor similarity. The Gaussian, representing the second RT component, was assumed not to vary with targetdistractor similarity, and the same parameters were used for all the fits.

We may now understand the relation between RT and similarity (Equation 1) in a more fundamental way. The exponential describes the frequency distribution of durations for a process that has a constant probability of completion at any moment in time. In this case, we assume that the process completed is the detection of the search target. Since variations in target-distractor similarity affected only the decay parameter of the exponential, we can assume that their effect was to change the moment-tomoment probability that the target would be found. Thus, the logarithmic rule stated above may be reformulated as an exponential relation between the probability of detecting a target and the similarity of that target to its surrounding distractors. The simplicity of this relationship suggests that search RT may reflect rather directly a basic property of form recognition in the search task, and we hope to use it in quantitative modeling of the detection process.

An extension of this analysis showed that a change in the relative rate of reinforcement for a correct detection affected RT in the same way, causing only a change in the exponential decay constant and thus a change in the momentary probability of response (D. S. Blough, 1989a). A given change in reinforcement changed the probability by a fixed amount, independently of variations in probability that were induced by changes in search display size and in target-distractor similarity. This outcome suggests that the processes determining these probabilities act independently. It would be interesting to develop a quantitative relationship between search RT and relative reinforcement probability through systematic variation of the latter through a wide range. Such a relationship would be comparable in character to Equation 1, and it would be useful in testing models of reinforcement.

The RT distributions for correct search detections, exemplified in Figure 7, contrasted remarkably with RT distributions of false alarms from a go/no-go generalization experiment (D. S. Blough, 1978). That experiment produced distributions of RTs to stimuli varying in similarity to an S+. An early RT mode was invariant across stimuli and reflected responses attributable to inattention. A second RT component showed that similarity to $\mathrm{S}+$ affected the chance that a trial would contain a response but did not affect the momentary probability of that response. These results have been used to exemplify the fastguess model of detection (e.g., Luce, 1986, p. $223 \mathrm{ff}$ ); a similar analysis using form stimuli might be productively conjoined with the results reviewed earlier. For present purposes, these data underline the interlocking role of attention and stimulus similarity in controlling discrimina- tive behavior. We turn next to consider more fully the role of attention in form perception and visual search.

\section{ATTENTION}

The research described so far specifies relationships among physical characteristics of complex forms and their detectability and suggests ways in which the perceptual system further analyzes such information. This section turns to studies of attention, which we define as a limitedcapacity cognitive resource that highlights selected aspects of a visual scene. A few examples have already entered our discussion: Sensitivity to particular aspects seems to wax and wane without experimental intervention (D. S. Blough, 1993b); RT distributions reveal a mode that probably reflects the pigeon's overall attentional state (D. S. Blough, 1989a); the typical rise in RT with display size may indicate serial redirections of attentional focus to different parts of the search screen.

The literature on attention is vast and includes a great many theoretical views, but there is substantial agreement as to certain basic ideas. It is common, for example, to distinguish between parallel and serial processes and their rough correspondence to the absence or presence of an attentional load (e.g., Hoffman, 1979; Schneider \& Shiffrin, 1977; Treisman \& Gelade, 1980). Variations in targetdistractor similarity have proven especially useful in establishing such distinctions. Our initial search studies centered on the manipulation of attentional load and related the findings to conceptualizations from human studies.

Other accepted ideas concern the focus of attention. In search, for example, experimental manipulations may direct attention toward a particular location or target. Here, the object of attention is defined by an improvement in discriminability while physical similarity is constant. Further pigeon search studies emphasized tasks that strongly engaged attention and suggested ways in which its focus could be directed. Such operations, known as priming, relate not only to human attentional theory (e.g., Posner \& Snyder, 1975) but also to work in animal foraging and to some human sensory data (Ball \& Sekuler, 1981; Davis, Kramer, \& Graham, 1983).

\section{Attentional Load}

Considerable research and theory have emphasized distinctions between tasks that do and do not impose an attentional burden. When discriminations are relatively easy and memory demands are low, performance appears to be nonattentive or automatic - that is, its efficiency is relatively independent of the number of subtasks to be accomplished. In visual search, the near absence of a display-size effect is a frequently used diagnostic. The searcher seems to process all display items simultaneously or in parallel; thus, search RT does not rise with the number of items in a display. A widely known example, mentioned above, is the case where the target can be 
identified by a distinctive feature (Treisman \& Gelade, 1980). More difficult tasks impose attentional demands and thus limit the number of subtasks that can be accomplished simultaneously. The attentive searcher seems to process various regions of the display sequentially, leading to a display-size effect and to the conclusion that search is serial or controlled. This outcome is seen in Treisman and Gelade's results for targets defined by conjunctions of features; because such targets share features with distractors, discriminations are more difficult. While some models stress strong distinctions between processes associated with parallel and serial search (Hoffman, 1979; Schneider \& Shiffrin, 1977), recent views (e.g., Wolfe, Cave, \& Franzel, 1989) propose more complex interactions.

In pigeon search, it is convenient to manipulate task difficulty by varying target-distractor similarity and then to assess attentional demands via the display-size effect. Several of our pigeon studies replicated basic results from human experiments. Figure 8 illustrates the role of perceptual variables in fixed-item search. The data in the left panel are from an early experiment that used an oscilloscope display (D. S. Blough, 1979); the results on the right are from a study using the touch-screen equipment described above (P. M. Blough, 1984). Both sets of functions suggest serial search with inspection time per item increasing with target-distractor similarity. This example does not show a flat display-size function presumed to indicate parallel search. Figure 11 below does illustrate this outcome, which occurred when the target was a very distinctive filled form among alphanumeric characters. In humans, parallel search may also be indicated when display sizes are relatively small and the subjects are well practiced (Shiffrin \& Schneider, 1977; Teichner \& Krebs, 1974).

An interesting exception to the usual display-size effect occurred with odd-item search (D. S. Blough, 1989c). Here, the slope of the function was reversed-that is, RT

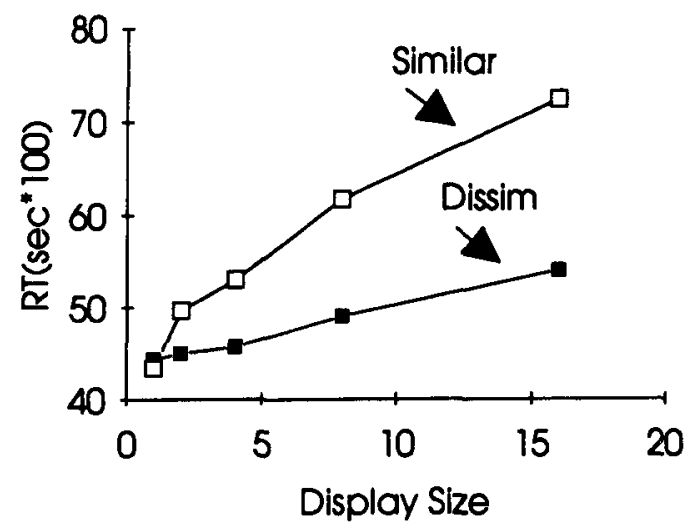

decreased with display size. This finding, along with some transfer data and results of Cook (1992), suggests that pigeons, like humans, are sensitive to configural influences. For example, large displays with many identical distractors may generate more easily discriminated configurations that enhance target distinctiveness and reduce attentional load.

The serial-parallel distinction may also apply to memory search, required when a random one of several possible targets appears in a given display. The well-known increasing relation between target-set size and RT (Sternberg, 1966) is most characteristic of tasks that rely on working memory - that is, when the memory set changes from trial to trial. When items in a target set are constant over trials, the increasing relation between set size and RT is evident when target-distractor similarity is high and when the searcher is relatively unpracticed (Shiffrin \& Schneider, 1977). With fewer task demands, a flat function is seen; Shiffrin and Schneider suggested that practice reduces attentional demands and thus permits automatic processing to emerge.

Two pigeon search studies varied target-set size in search tasks requiring rather difficult discriminations. The composition of the target set was fixed over trials (D. S. Blough, 1979; P. M. Blough, 1984). Neither of these experiments revealed set-size effects. Given the extensive practice required in advance of final data collection, these outcomes are consistent with Shiffrin and Schneider's (1977) analysis. Recently completed work in our lab (Vreven \& Blough, 1996) documents the emergence of such practice effects more fully.

\section{Attentional Priming}

In this section, we consider studies that vary attentional focus. In visual search, attention improves detection when it is directed toward a particular target or location. The abundant literature on this topic describes several

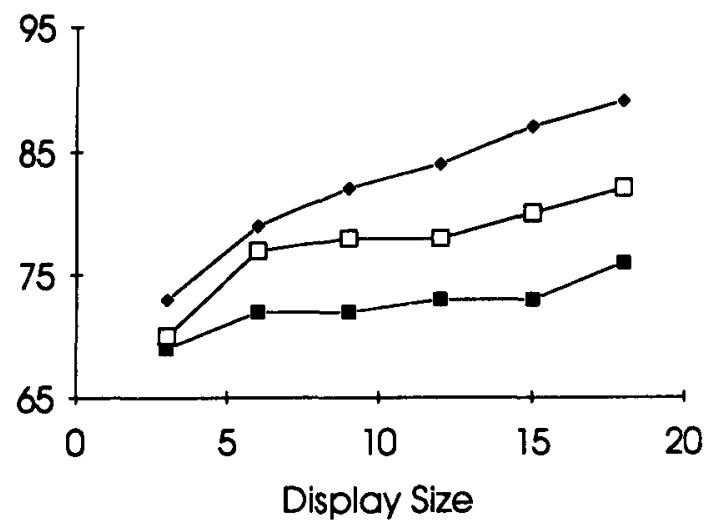

Figure 8. Display-size effect in two search procedures. The abscissa represents total number of items, including a single target, in a search display. Similarity refers to the target-distractor relationship. Left panel: Display appeared on an oscilloscope screen, and responses were sensed by a photocell mounted on the pigeon's beak (D. S. Blough, 1979, Experiment 1). Right panel: Display appeared on a video monitor, and responses were sensed by a touch frame. Lower, intermediate, and upper functions are associated with low, medium, and high levels of similarity, respectively (P. $M$. Blough, 1984, Experiment 3). 
operations that seem to direct attention, and we refer to these as priming events. Under conditions that favor parallel search, as in highly discriminable displays, priming should theoretically be ineffective. When search is attentive, benefits resulting from focus should be accompanied by costs in that detection of unattended items worsens.

Most priming events provide information about the identity or location of the target to appear on the forthcoming trials. Perhaps the most straightforward example is the use of a single cue just before trial onset (Beller, 1971; Posner \& Snyder, 1975). Trial sequences can also provide information; for example, when trial sequences randomly mix values along a psychophysical continuum, discrimination is poor relative to conditions that present a single value within a trial block (Ball \& Sekuler, 1981; Davis et al., 1983).

Related findings have emerged from studies of avian foraging. Much of this work has used naturalistic tasks in which a bird selects food items from a patch including multiple food types (e.g., Bond, 1983; Langley, Riley, Bond, \& Goel, 1996; Reid \& Shettleworth, 1992). A central finding replicates Tinbergen's (1960) field studies, showing that birds select abundant prey with a probability that exceeds their relative frequency in the patch. Tinbergen accounted for his findings by postulating a filterlike search image, induced by repeated encounters with a given prey. This hypothesis has prompted a variety of experiments that relate to attentional priming. In some interesting laboratory work, for example, Pietrewicz and Kamil (1979) used same-target ("blocked") and mixedtarget trial sequences in a study that required jays to discriminate among photographically displayed moth types; as in the psychophysical work mentioned above, the jays were faster and more accurate during the blocked sequences.

Our work on attentional priming has been guided by the view that information about a forthcoming target's identity activates a target representation in advance of an en- counter. Such activation could speed a process by which displayed targets are compared with representations drawn from memory. The advance information can take several forms, including single informative cues, predictable trial sequences, or differential target probabilities within a session.

Initial studies used both single and sequential cues to target identity in multiple-target search paradigms. Figure 9 illustrates these approaches. Some of these experiments (left panel) have replicated Pietrewicz and Kamil's (1979) effect in our search procedure - that is, pigeons' RTs were shorter within blocked sequences than they were within mixed sequences (P. M. Blough, 1989, 1991). Others (right panel) replicated Beller's (1971) work, showing that pigeons recognized target letters faster when the trial was preceded by a symbolic visual cue signaling the target's identity (P. M. Blough, 1989, 1991). In a related procedure, D. S. Blough (1993a, Experiment 2) showed speeded search when target location was signaled by within-trial context; in that work, right and left screen halves had unique associations with particular distractors in a homogeneous array.

Finally, in a design that more closely resembles natural variations in prey abundance, several experiments considered the priming effectiveness of relative target frequency. In this work, one member of a target set occurred with a relatively high probability within a session; however, the identity of the frequent target varied between sessions. Search speed was relatively high for the more probable target (P. M. Blough, 1991, 1992, 1996) or for more probable target-distractor pairs (D. S. Blough, 1993a). Figures 11 and 14 below illustrate RT data from this method. Choice procedures have also revealed the target-probability effect: When probe trials displayed two targets, the pigeons were more likely to peck at the form associated with the higher probability during a preceding sequence (P. M. Blough, 1992, Experiment 3). Reid and Shettleworth (1992, Experiment 3) obtained
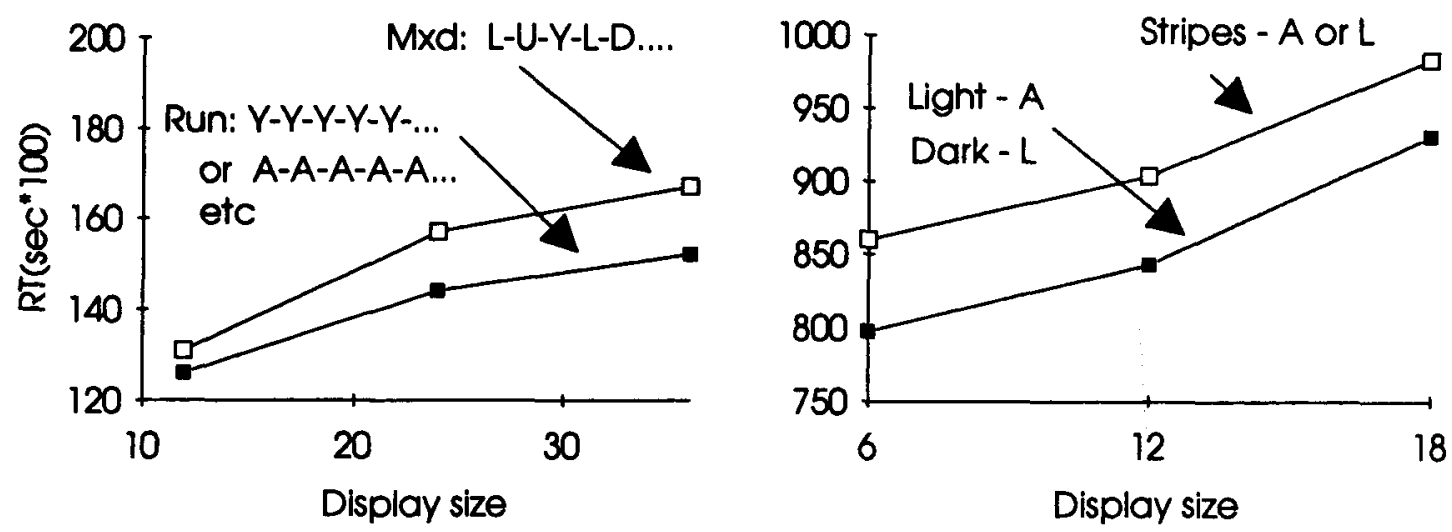

Figure 9. Two types of priming operations and associated data. Left panel: Priming by same-target sequences (runs). In the mixed sequence, the target to appear on a given trial was selected at random from the multiple-target set. Both types of sequence were made up of 48 trials (P. M. Blough, 1989, Experiment 2). Right panel: Priming by symbolic cues, appearing as borders on each side of the display screen. Light and dark borders preceded specific targets, whereas the striped border could signal either target (P. M. Blough, 1989, Experiment 1). 
similar results in an experiment that used natural grains as targets. Both studies conceptually replicate Bond's (1983) work, which assessed choice among grains whose relative numbers within a patch were systematically varied.

Like most real-world stimuli, natural foods of a given type vary in appearance, and it is reasonable that a primed representation should refer to a class of stimuli rather than an individual exemplar. In a human experiment, Rosch (1975) found that color names facilitated performance on a color-matching task even when the named color varied in saturation or lightness. Reid and Shettleworth (1992) suggested that a search image represents a class of prey identified by a shared feature. However, in our laboratory, we failed in an attempt to tune attention to a class of forms characterized by a particular feature (D. S. Blough, 1993a, Experiment 1). In a feature-relevant condition, a class of probable targets could be identified by a line or, in another phase, a gap. While RTs were lower for more probable target class, a subsequent control showed similar facilitation when the probable target did not possess a distinguishing feature. This outcome is reminiscent of some of the early pigeon-concept work, where the birds appeared to have memorized a large set of exemplars (Vaughan \& Greene, 1983). It is reasonable that the variability inherent among natural grains and their backgrounds would favor more versatile search images than is the case with artificial symbols of fixed appearances.

Costs and benefits. If priming engages a limitedcapacity resource, it should not only improve the detectability of the primed item but it should also interfere with the detection of alternative targets. Posner and Snyder (1975) defined benefits and costs of primes with reference to a control condition using an ambiguous cuethat is, benefits are seen when primed performance is more efficient than the ambiguous control, and costs are seen when it is less efficient. The blocked-mixed and the symbolic-cue procedures provided this control and established benefits by this criterion. To probe costs, a vari-

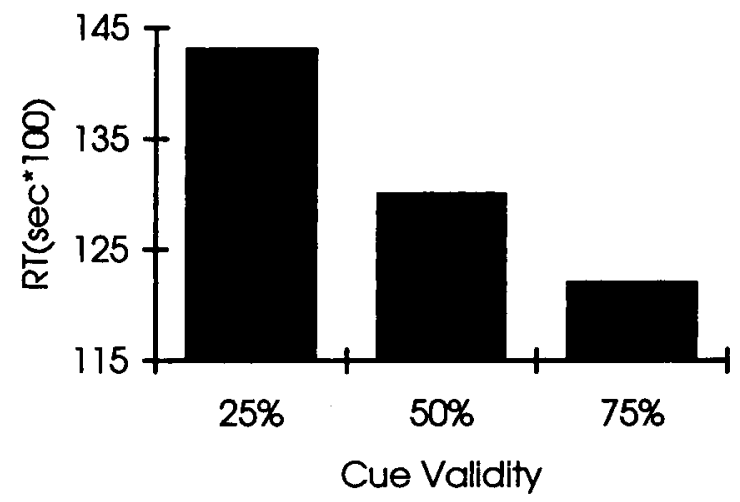

ation of the symbolic priming procedure reduced the validity of the informative cues to $75 \%$; thus, $25 \%$ of the trials were miscued (P. M. Blough, 1989, Experiment 3). Figure 10 (left panel) shows the outcome and illustrates attentional costs-that is, RTs were higher following miscues than they were following ambiguous visual signals. A frequency-priming study (D. S. Blough, 1993a) exemplifies costs in a different way. RTs associated with a target increased when its probability was reduced, relative to an equal-probability baseline (Figure 10, right panel). Benefits were not significant in this example and may have been obscured by floor effects. An analysis of costs and benefits in the blocked-mixed paradigm revealed the expected RT pattern but did not establish significant costs (P. M. Blough, 1991).

Perceptual factors. We referred above to variations in attentional requirements associated with particular tasks. When attention is not needed, as when a very dissimilar target pops out, priming should be ineffective. Tinbergen (1960) and others have suggested that foragers adopt search images only in cryptic conditions. Bond's (1983) laboratory study used two levels of concealment; his pigeons overselected the more abundant of two grains in the cryptic condition but not in the conspicuous condition. This outcome is roughly consistent with the idea that easily detected targets bypass an attentional filter.

A different line of reasoning emphasized the costs associated with search-image formation. Guilford and Dawkins (1987) noted that a search image could have maladaptive consequences if it caused the forager to miss easily detected prey. As an alternative to attentional accounts, they proposed that foragers adjust their search speed to the conspicuity of an abundant prey. This strategy could be more beneficial than a perceptual filter, because it would permit detection of alternative prey that were equally or more conspicuous.

We have explored Guilford and Dawkins's (1987) hypothesis and have attempted to define the cryptic require-

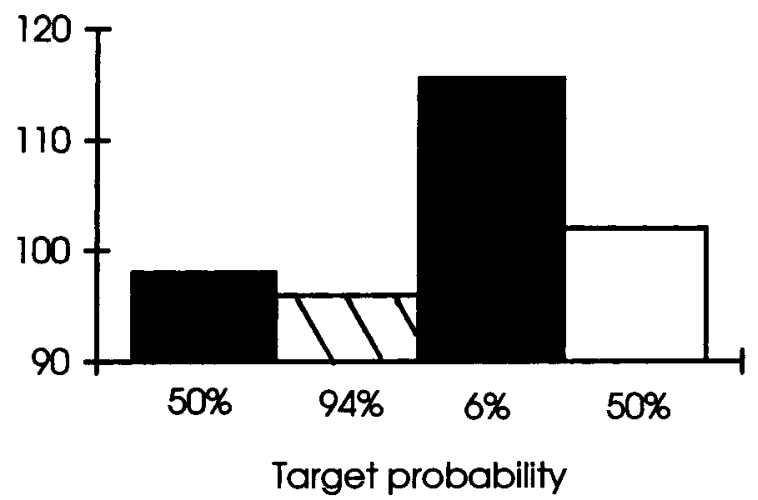

Figure 10. Costs of priming. Left panel: Data from the symbolic-cue procedure as in Figure 9 (right panel). However, on $25 \%$ of the trials, the usual association between targets and light or dark borders was reversed (cue validity $=25 \%$ ). The difference between the RTs associated with the $25 \%$ and $50 \%$ conditions was significant, indicating priming costs (P. M. Blough, 1989, Experiment 3). Right panel: RTs from a frequency-priming study that used several target-distractor combinations in odd-item search. Probabilities associated with separate sets of these combinations were equal (left and right segments) or unequal (center segment). Costs are seen in the significant $R T$ elevation for the less frequent set after the unequal condition was introduced (D. S. Blough, 1993a, Experiment 1). 
ment more fully. As expected, with a very low perceptual load (display size of one), target probability appears to have no effect on detectability (D. S. Blough, 1992; P. M. Blough, 1989, Experiment 4). Nonetheless, symbolic-cue and frequency priming are effective over a wide range of display sizes (5-36 items), and the magnitude of priming does not appear to change over this range (P. M. Blough, 1989, Experiment 1; P. M. Blough, 1991). Also, manipulations of target-distractor similarity yielded no evidence for an interaction between concealment and priming magnitude (P. M. Blough, 1989, Experiment 4; P. M. Blough, 1992). Indeed, in the latter study, frequency priming was effective in a display that seemed to be processed in parallel. Figure 11 illustrates this finding. The highly detectable "heart" (hrt) target yielded a flat display-size function (left), yet frequency affected RT to this salient form (right). These data suggest a dissociation between display-size and priming criteria for assessing attentional load. They also challenge Guilford and Dawkins's (1987) search-rate hypothesis and modify as well the cryptic requirement for search images, suggested in the foraging literature.

Memory factors. Most nonhuman studies of priming have used fixed memory sets of two items (e.g., two moth species, Pietrewicz \& Kamil, 1979; two grain types, Bond, 1983; two forms, P. M. Blough, 1989). Activation accounts imply that the priming event enhances the accessibility of one of these items. However, results from a recent pigeon study (P. M. Blough, 1991) suggest conditions in which the benefits of advance information diminish with experience. This possibility first emerged as an annoyance; procedures using the blocked-mixed paradigm initially showed the priming effect at a target-set size of three. However, in follow-up work, using the same birds, the effect faded and then returned when three new target items were added. Perhaps, with extended training, the pigeons adopt an automatic memory-search modethat is, the birds may come to address representations of all members of the target set in parallel during the mixedtrial sequences, just as they seemed to in sessions that mixed targets throughout (P. M. Blough, 1989). Vreven and Blough's (1996) recently completed research in our lab, mentioned above, documents reductions in blockedmixed priming with experience.

\section{Origins of Attentional Modifiers}

A variety of events modify attention, and they do not necessarily have common origins. We have emphasized expectancy priming, but attention may be captured by bottom-up sources, such as distinctive features, and by unknown sources implied by D. S. Blough's (1993b) data on attentional fluctuations. In another setting, work by D. S. Blough (1969) documented attentional changes brought about by extended practice. This work used stimulus generalization, training birds to respond in the presence of a single combination ( $S+$ ) of light wavelength and sound frequency. Additional, negative, stimuli were drawn from both dimensions. Figure 12 (left segments) illustrates the resulting generalization gradients along both dimensions. During subsequent experimental phases, the birds practiced this discrimination along just one of the continua and again experienced the two-dimensional test. The additional panels in Figure 12 show these results. Practice resulted in a steeper generalization gradient for the practiced dimension and a flatter gradient for the nonpracticed dimension. This pattern of results is consistent with a practice-induced shift of attention to the practiced dimension and away from the alternative dimension. These training effects, while conceptually similar to those of priming, are considerably more long-lasting, extending over hours rather than seconds.

Expectancy learning. Associative learning must play a role when arbitrary cues forecast target identity and thus build "expectancies." Indeed, priming emerged only after considerable training, and it diminished with increasing delays between cue and search display (P. M. Blough,
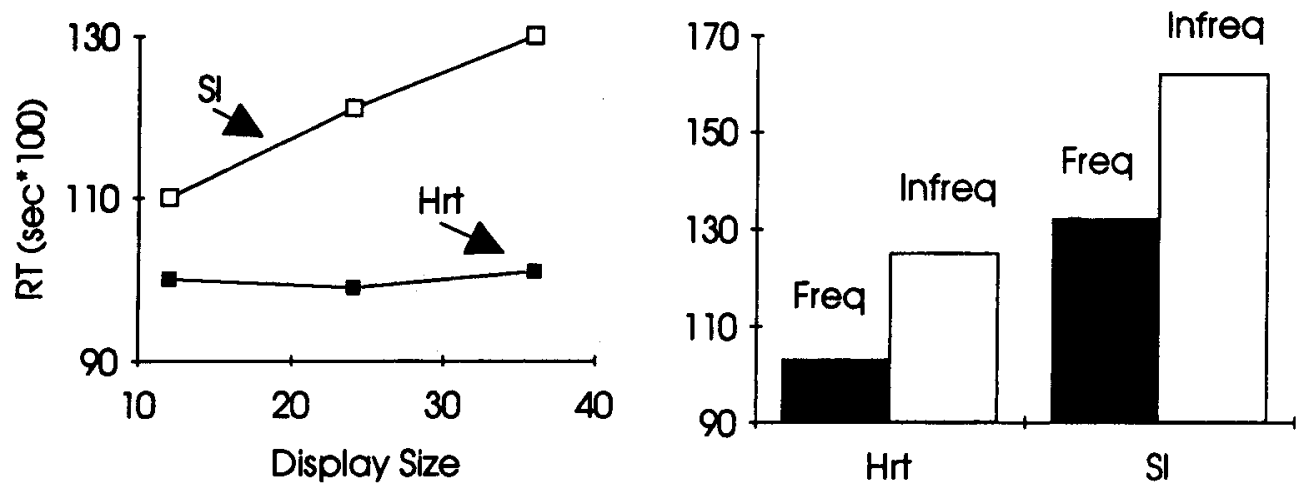

Figure 11. Effects of frequency priming on salient and less salient targets. Left panel: Display-size functions associated with filled heart (hrt) and backslash (sl) targets among alphanumeric distractors. Right panel: Effects of relative frequency in two session types corresponding to the two pairs of bars. 


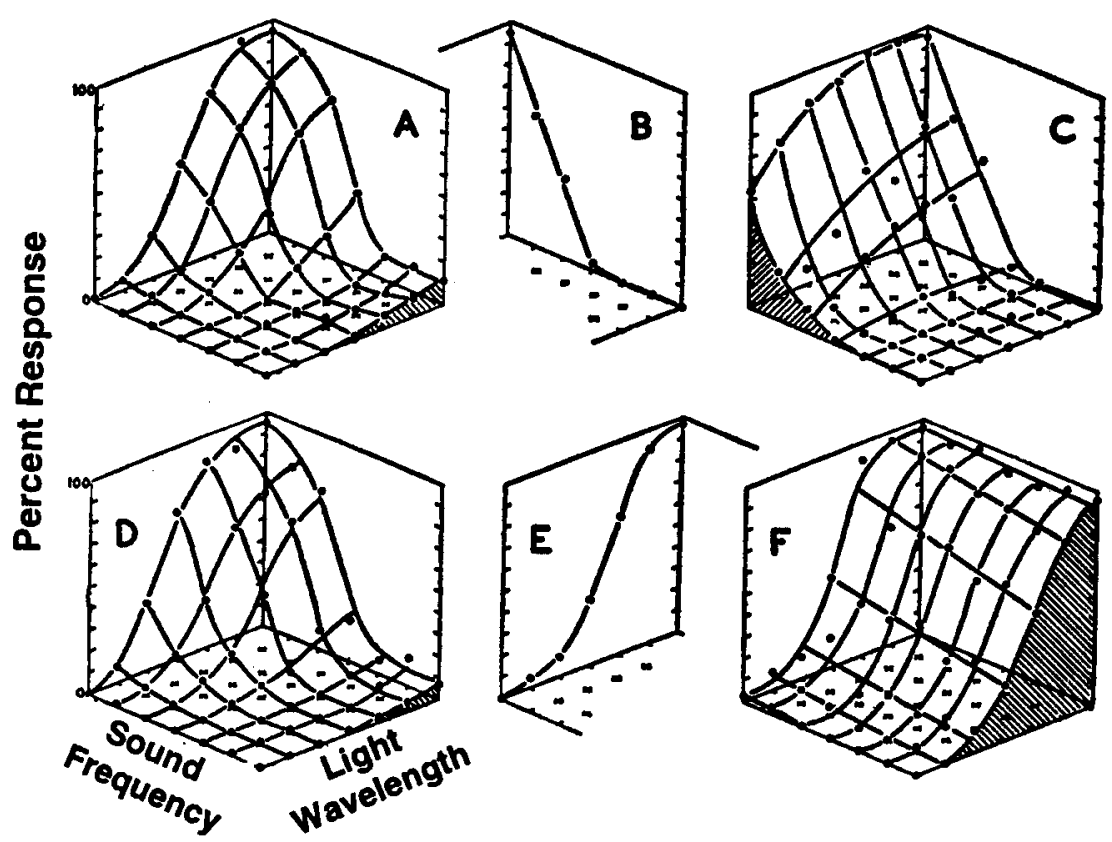

Figure 12. Effects of training on generalization to visual and auditory dimensions. Auditory and visual stimuli occurred together on each trial. (A) Baseline procedure in which reinforcement was contingent on responses to a single sound frequency and light wavelength combination. (B) Visual stimulus was constant at the reinforced value, and the auditory stimulus varied as in baseline. (C) Baseline procedure on the session just following the procedure in panel $B$. Panels D, E, and F follow the same plan, but the auditory stimulus was constant at its reinforced value in Panel E (D. S. Blough, 1969).

1989, 1991). The priming effectiveness of display context (D. S. Blough, 1993a) also implies acquired associations between unrelated forms.

On the other hand, priming by target repetition could be attributed to an unlearned process. For example, the representation of a target on a given trial could carry over to the next trial in the form of a sensory trace. A number of human studies implicate such effects; Kirby (1980) showed that, within a random sequence, quick repetition of the just-preceding trial facilitated detection. A model proposed by Bond (1983) is consistent with those data. According to this view, a second encounter with a given prey, if it occurs almost immediately, triggers a focused attentional mode.

A recent study (P. M. Blough \& Lacourse, 1994) attempted to disentangle such bottom-up influences from top-down learning effects. We reasoned that a sensory trace of a target might facilitate search for the same target regardless of any predictive relationship - that is, search would be more rapid when a trial repeated the justprevious target, even when the repetition occurred by chance during a random sequence. On the other hand, learning-based priming would require the subject to experience predictive relationships among trials. This work used blocked sequences to create such relationships. In a predictable condition, a given target, drawn from a set of 11 , was repeated for exactly three trials, and target identity then changed. In a second condition, target sequences were random. A 2-sec warning signal separated the threetrial sets and a 1-sec intertrial interval separated trials. Analysis applied to the random condition selected trial sets within which same-target sequences occurred by chance. Figure 13 illustrates the findings. The overall drop in RT was probably attributable to disruption from the warning signal. However, the decline was more rapid and final RTs were lower during the predictable condition than

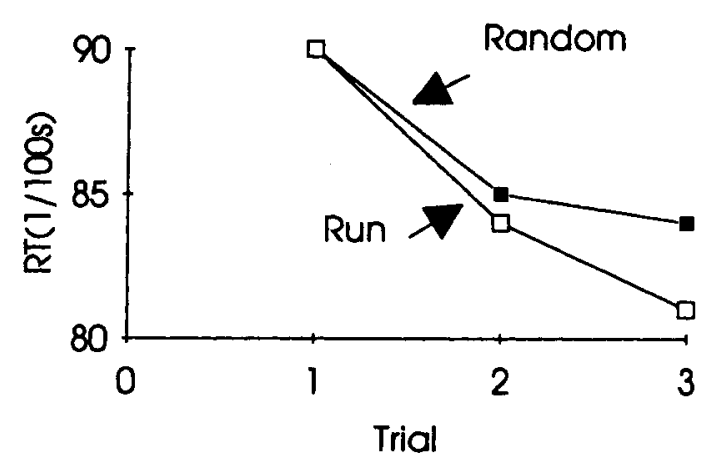

Figure 13. RTs associated with predictable and unpredictable same-target sequences. The abscissa represents the three trials that made up a sequence. Sessions during the runs condition were made up entirely of same-target sequences; in the random condition, RTs are from same-target sequences that occurred by chance during a random target-selection method (P. M. Blough \& Lacourse, 1994, Experiment 2). 
during the random condition. Similar outcomes occurred when location repetition was tested in the same fashion.

Thus, the blocked-trial effect seems to require predictability, and that, in turn, suggests that the pigeons learn the pertinent experimental rules. One such rule could be that the next target will most likely have the same identity as the current one. In blocked-mixed designs, this principle would provide valid information within a same-target sequence and costly miscues within a mixed sequence. However, if the pigeons discriminate blocked from mixed sequences, miscues should not occur; in that case, the associated priming effect would reflect only the benefits of an informative cue. A related interpretation comes from work in visual psychophysics where the descriptors' "certainty" and "uncertainty" have been applied to the difference between same-stimulus and mixed-stimulus sequences (Ball \& Sekuler, 1981; Davis et al., 1983).

Recent work has considered the role of experience in priming by relative frequency (P. M. Blough, 1996). Figure 14 illustrates the course of the frequency effect; these data are from a study that replicated P. M. Blough's (1996) work, but with a smaller target set. The functions show RTs during two segments of a 1,200-trial session. An initial 600-trial phase presented one of the two targets with a probability of $90 \%$ and the alternative target on the remaining $10 \%$ of the trials. The identity of the frequent target changed unpredictably from session to session. As shown, RTs declined over trials, although less so for the less probable target. During a second 600 -trial phase, the two targets occurred with equal probabilities; here, RTs converged and continued to decline slightly. Sequential analyses, applied to P. M. Blough's (1996) data, suggested that RTs were unaffected by the targets on just-preceding trials. It is unlikely, then, that the target-probability ef- fect is attributable to expectancies based on very recent experience.

In a speculative interpretation, P. M. Blough (1996) suggested that the birds evaluated target probabilities over a relatively large trial window. Such sampling would construct an informative representation that, over trials, would increasingly approximate actual target probability, and final RT levels would depend on that value. This interpretation is consistent with the relatively large initial RT decline for the more probable target; it is also consistent with the convergence of RTs during the equalprobability condition. However, control entirely by a probability-based representation would have led to a rise in RTs for the initially frequent target in the second phase when its likelihood decreased.

An alternative interpretation stresses the role of frequency without any representation of probability. In this view, conceptually related to a model suggested by Logan (1988), RT declines simply as a result of exposure to a given event. The frequency effect would result from differences in total exposures per session rather than probability, and it should disappear as asymptotic RTs are approached. This interpretation better accounts for the continuing, but slow, RT decline during the equalprobability phase (Figure 14, right segments). However, the data so far do not fully distinguish between probabilitybased and frequency-based control of RTs. As seen in Figure 14 (left segments), there was relatively little exposure to the infrequent target and thus insufficient information to estimate asymptotic performance in this condition. Should the frequency effect disappear over extended exposure to all targets, there would be an intriguing parallel to practice-induced reductions in the blocked-trial effect (P. M. Blough, 1991).

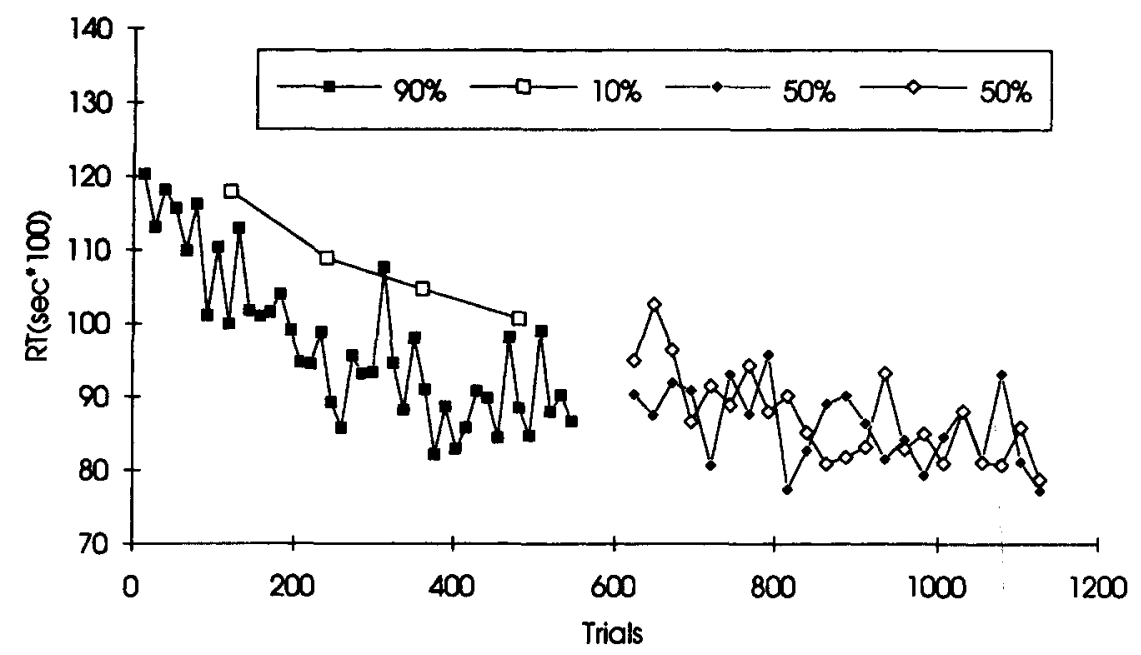

Figure 14. Course of RTs over an unequal-probability condition (left segments) and a second equal-probability condition (right segments). The second condition followed the first within each session; the filled symbols represent the formerly frequent target, and the open symbols represent the formerly infrequent target. Each data point summarizes 12 trials with a given target; its position with respect to the abscissa reflects point with the session when 12 such trials had accumulated. Data are averaged over 16 sessions and 3 birds (P. M. Blough, 1995). 
The role of reinforcement. Reinforcement has surprisingly weak effects on expectancy learning. Further study of priming by relative frequency determined that RT reductions, such as those in Figure 13, occur in the absence of reinforcement. In particular, the differential patterns associated with frequent and infrequent targets persisted during extinction sessions. Because the identity of the frequent target changed between sessions, there could not have been carryover from prior experiences with differential reinforcement (P. M. Blough, 1996, Experiment 2). Nonetheless, as we will show, associating a target with differential reinforcement also reduces RT for that target. We may conceptually distinguish these effects by assuming that priming speeds the detection of a target, whereas differential reinforcement might affect the speed of response to a target after it has been detected. In that case, reinforcement effects would be relatively independent of the conditions that affect detection.

Evidence for such a dissociation comes from several sources. Two studies showed reinforcement effects that could not be explained by priming. In the first (D. S. Blough, 1989a), either of two targets could appear with equal probability under several distractor conditions. Increasing the reinforcement probability associated with one of the targets from $10 \%$ to $30 \%$ markedly increased the search RT to the other target. This effect was just as pronounced when there were no distractors and thus no attentional load, as it was in the most difficult search condition. The second study also used two targets, each presented alone on a blank field (D. S. Blough, 1992). It showed a powerful effect on RT when the probability of reinforcement (reinforcements per trial with a given target) differed for the targets. On the other hand, overall frequency of reinforcement did not affect search when target probabilities were equal; RTs seemed unaffected when one target appeared 19 times as often as the other, and responses to it received (in absolute terms) 19 times as much reinforcement. This condition would have been expected to produce a strong priming effect had there been distractors.

Taken together, these two studies confirm an effect of differential reinforcement probability on RT and strongly suggest its dissociation from priming. There is considerable independent evidence that reinforcement probability is the key variable controlling response strength in this sort of situation (see Williams, 1988), and it is likely that RT reflects response strength to targets once found. Frequency priming seems to belong to a class of attentional manipulations that affect a target's detectability; its occurrence under several conditions of equal reinforcement probability (e.g., D. S. Blough, 1993a; P. M. Blough, $1992,1996)$ and in the absence of reinforcement (P. M. Blough, 1996) is also consistent with the suggested dissociation.

Of course, this analysis does not rule out indirect effects of reinforcement. The idea that attention itself can be reinforced is familiar (see, e.g., Hall, 1991). While reinforcement may not guide the detection process itself, in pigeon search, it is probably crucial to the expectancy learning that underlies priming.

\section{SUMMARY}

We have reviewed explorations of the ways in which pigeons process information about visual form. Most of the studies used visual search, and the time taken by a bird to find a target displayed on a computer screen, the search RT, proved to be a particularly informative variable. Partly because search RTs are widely used in experiments with humans, our pigeon data could often be related in a common conceptual framework to data from humans. This framework incorporated such familiar ideas as features and dimensions, parallel and serial processing, and the distinction between bottom-up operations driven largely by stimulus variables and top-down expectancy and learning effects.

Forms were characterized primarily in terms of their similarity to one another, with similarity measures derived from search RT and accuracy data. Similarity classifications of alphabetic letters gave insights into the appearance of these simple forms to pigeons and also provided scaled stimulus materials for subsequent research. Patterns of pigeon and human letter similarities bore a striking resemblance to one another, suggesting that processing might be similar for the two species. Letter groups could be largely characterized by the presence of common features, such as "open center" and "open right," which suggested that such features might be extracted in the search process. Subsequent studies sought more definitive evidence of feature analysis, and a new covariation method provided supporting data. However, a study of search asymmetries replicated several known features in human subjects but failed to show the same features in pigeons. This difference raised, among other things, the interesting question to what extent a "feature" is defined by selective exposure and past practice with forms.

The dimensional analysis of similarity also yielded interesting results. MDS suggested that different stimulus aspects sometimes act with relative independence (separable dimensions) and sometimes interact (integral dimensions), an observation familiar from experiments with humans and again suggesting common modes of processing across species. A series of experiments with several form sets established an exponential relationship between the similarity of form pairs and search RT involving those pairs. Search RT distributions proved to be very well fit by a convolution of Gaussian and exponential distributions, with the effects of form similarity carried by a single parameter, the exponential decay constant. Together, these findings enabled us to state an exponential function relating the probability of detecting a target to the similarity of that target to its surrounding distrac- 
tors. The simplicity of this relationship suggests that search RT may rather directly reflect basic properties of a form recognition process.

In pigeons, as in humans, attention is influenced by both past experience and the properties of the stimulus field. Unless targets and distractors are highly dissimilar, search time rises with display size, a pattern that is said to characterize serial search - that is, it would result if various parts of the display were analyzed sequentially with focused attention. The effect is enhanced when targets and distractors are similar, suggesting increased processing time per display item. Using similarity estimates based on our scaling of letters, we replicated the interactive relation between display size and similarity in several experiments, but we identified only one condition in which a very dissimilar target induced the flat RT function that characterizes pop-out, thought to reflect parallel processing. As a whole, the pattern of results suggested that attentional modulation of search in pigeons is understandable within the conceptual framework developed from experiments with humans.

Other research modified attentional focus through priming, where the subject received advance information about the identity of an upcoming target. Valid primes speeded the detection of a target, relative to detection following ambiguous or misleading information. Specifically, detection was faster if a given target's identity was signaled by (1) a brief predictive cue that appeared just before the trial, (2) trial sequences regularly associated with the target, and (3) a high probability that the given target would appear, relative to other possible targets. Other results implicated the role of expectancy in these effects; in particular, the facilitative effects of sequential cues seem to arise neither from stimulus-driven representations carried over from very recent trials nor from differential reinforcement associated with the primed target. Overall, our data suggest that pigeons can learn predictive relations among stimuli; relations that convey relevant information then tune attention to probable targets.

We began this review by remarking on the ease with which one assumes that birds see the world as we do. Yet this is simply a guess until appropriate experiments are done. Some of our experiments, together with those from other laboratories, have begun to confirm this guess in simple cases. This should not disappoint us, since, if pigeons classify forms as we do or their attention is guided as ours is, these observations suggest a remarkable generality of biological solutions to common perceptual problems, and they open a new venue in which to explore these solutions. Unique results from pigeons, such as the failure of search asymmetry, are informative in a different way. Do they suggest unexpected influences (perhaps human search asymmetries arise from past experience with forms), or do they point to interesting species differences? Finally, there are phenomena and relationships that appear to be fundamental but that may be explored most readily in nonhuman animals. One such, perhaps, is the relation of similarity to search $\mathrm{RT}$, which was derived from a gigantic number of experimental trials; another might be the effect on form perception of carefully controlled past experience. We anticipate with interest those future experiments that will further unfold the perceptual and conceptual worlds of creatures different from ourselves.

\section{REFERENCES}

Allan, S. E., \& Blough, D. S. (1989). Feature-based search asymmetries in pigeons and humans. Perception \& Psychophysics, 46, 456-464.

Ball, K., \& SeKuler, R. (1981). Cues reduce direction uncertainty and enhance motion detection. Perception \& Psychophysics, 30, 119-128.

BELLER, H. K. (1971). Priming: Effects of advance information on matching. Journal of Experimental Psychology, 57, 176-182.

BiedermaN, I. (1987). Recognition-by-components: A theory of human image understanding. Psychological Review, 94, 115-147.

Blough, D. S. (1969). Attention shifts in a maintained discrimination. Science, 166, 125-126.

BLOUGH, D. S. (1972). Recognition by the pigeon of stimuli varying in two dimensions. Journal of the Experimental Analysis of Behavior, 18, 345-367.

BLough, D. S. (1978). Reaction times of pigeons on a wavelength discrimination task. Journal of the Experimental Analysis of Behavior, 30, 163-167.

BLOUGH, D. S. (1979). Effects of the number and form of stimuli on visual search in the pigeon. Journal of Experimental Psychology: Animal Behavior Processes, 5, 211-223.

Blough, D. S. (1982). Pigeon perception of letters of the alphabet. Science, 218, 397-398.

BLough, D. S. (1985). Discrimination of letters and random dot patterns by pigeons and humans. Journal of Experimental Psychology: Animal Behavior Processes, 11, 261-280.

BLOUGH, D. S. (1986). Odd-item search by pigeons: Method, instrumentation, and uses. Behavior Research Methods, Instruments, \& Computers, 18, 413-419.

BLough, D. S. (1987). [Search among forms of different sizes]. Unpublished raw data.

Blough, D. S. (1988). Quantitative relations between visual search speed and target-distractor similarity. Perception \& Psychophysics, 43, 57-71.

BLOUGH, D. S. (1989a). Contrast as seen in visual search reaction times. Journal of the Experimental Analysis of Behavior, 52, 199-211.

BLOUGH, D. S. (1989b). Features of forms in pigeon perception. In W. Honig \& G. Fetterman (Eds.), Cognitive aspects of stimulus control (pp. 263-277). Hillsdale, NJ: Erlbaum.

BLOUGH, D. S. (1989c). Form similarity and categorization in pigeon visual search. In M. C. Commons, S. Kosslyn, \& R. J. Herrnstein (Eds.), Quantitative analyses of behavior, VIII (pp. 129-143). Hillsdale, NJ: Erlbaum.

Blough, D. S. (1991). Perceptual analysis in pigeon visual search. In G. Lockhead \& J. Pomerantz (Eds.), The perception of structure. Washington, DC: American Psychological Association.

BLough, D. S. (1992). Effects of stimulus frequency and reinforcement variables on reaction time. Journal of the Experimental Analysis of Behavior, 57, 47-50.

BLOUGH, D. S. (1993a). Effects on search speed of the probability of target-distractor combinations. Journal of Experimental Psychology: Animal Behavior Processes, 19, 107-120.

BLough, D. S. (1993b). Reaction time drifts identify objects of attention in pigeon visual search. Journal of Experimental Psychology: Animal Behavior Processes, 19, 107-120.

Blough, D. S., \& Blough, P. M. (1990). Reaction time assessments of visual perception in pigeons. In W. C. Stebbins \& M. A. Berkley (Eds.), Comparative perception (Vol. 2, pp. 245-276). New York: Wiley. 
Blough, D. S., \& Franklin, J. J. (1985). Pigeon discrimination of letters and other forms in texture displays. Perception \& Psychophysics, 38, 523-532.

BLough, P. M. (1984). Visual search in pigeons: Effects of memory set size and display variables. Perception \& Psychophysics, 35, 344-352.

Blough, P. M. (1989). Attentional priming and visual search in pigeons. Journal of Experimental Psychology: Animal Behavior Processes, 15, 358-365.

BLough, P. M. (1991). Selective attention and search images in pigeons. Journal of Experimental Psychology: Animal Behavior Processes, 17, 292-298.

BLough, P. M. (1992). Detectability and choice during visual search: Joint effects of sequential priming and discriminability. Animal Learning \& Behavior, 20, 293-300.

BLough, P. M. (1995). [Effects of target probability]. Unpublished raw data.

Blough, P. M. (1996). Priming during multiple-target search: The cumulative effects of relative target frequency. Animal Learning \& Behavior, 24, 394-400.

BLOUGH, P. M., \& LaCOURSE, D. M. (1994). Sequential priming in visual search: Contributions of stimulus-driven facilitation and learned expectancies. Animal Learning \& Behavior, 22, 275-281.

BoND, A. B. (1983). Visual search and selection of natural stimuli in the pigeon: The attention threshold hypothesis. Journal of Experimental Psychology: Animal Behavior Processes, 9, 292-306.

BOND, A. B., \& RILEY, D. A. (1991). Searching image in the pigeon: A test of three hypothetical mechanisms. Ethology, 87, 203-224.

BorING, E. G. (1942). Sensation and perception in the history of experimental psychology. New York: Appleton-Century.

Burns, B., Shepp, B. E., McDonough, D., \& WiEner-Ehrlich, W. K. (1978). The relation between stimulus analyzability and perceived dimensional structure. In G. H. Bower (Ed.), The psychology of learning and motivation (Vol. 12, pp. 77-116). New York: Academic Press

Cook, R. G. (1992). Acquisition and transfer of visual texture discriminations by pigeons. Journal of Experimental Psychology: Animal Behavior Processes, 18, 341-353.

Corbetta, M., Shulman, G. L., Miezin, F. M., \& Petersen, S. E. (1995). Superior parietal cortex activation during spatial attention shifts and visual feature conjunction. Science, 270, 802-805.

Davis, E. T., Kramer, P., \& Graham, N. (1983). Uncertainty about spatial frequency, spatial position, or contrast of visual patterns. Perception \& Psychophysics, 33, 20-28.

Delius, J. D., \& Hollard, V. D. (1995). Orientation invariant pattern recognition by pigeons (Columba livia) and humans (Homo sapiens). Journal of Comparative Psychology, 109, 278-290.

Donovan, W. J. (1978). Structure and function of the pigeon visual system. Physiological Psychology, 6, 403-437.

DunN-RANKIN, P. (1983). Scaling methods. Hillsdale, NJ: Erlbaum.

Fujita, K., Blough, D. S., \& Blough, P. M. (1991). Pigeons see the Ponzo illusion. Animal Learning \& Behavior, 19, 283-293.

GARNER, W. R. (1974). The processing of information and structure. Hillsdale, NJ: Erlbaum.

GARNER, W. R. (1978). Aspects of a stimulus: Features, dimensions, and configurations. In E. Rosch \& B. B. Lloyd (Eds.), Cognition and categorization (pp. 99-133). Hillsdale, $\mathrm{NJ}$ : Erlbaum.

GRANDA, A. M., \& MAXWELl, J. H. (1979). Neural mechanisms of behavior in the pigeon. New York: Plenum.

GuILFORD, T., \& DAWKINS, M. S. (1987). Search images not proven: A reappraisal of recent evidence. Animal Behaviour, 35, 1838-1845.

Hall, G. (1991). Perceptual and associative learning. New York: Oxford University Press.

Heinemann, E. G., \& Chase, S. (1989). A quantitative model for pattern recognition by animals and people. In M. L. Commons, R. J. Herrnstein, S. M. Kosslyn, \& D. B. Mumford (Eds.), Quantitative analyses of behavior IX: Computational \& clinical approaches to pattern recognition \& concept formation (pp. 109-126). Hillsdale, NJ: Erlbaum.

HERRNSTEIN, R. J. (1979). Acquisition, generalization, and discrimination reversal of a natural concept. Journal of Experimental Psy. chology: Animal Behavior Processes, 5, 116-129.
Hoffman, J. E. (1979). A two-stage model of visual search. Perception \& Psychophysics, 25, 319-327.

JULESZ, B. (1981). Textons, the elements of texture perception and their interactions. Nature, 290, 91-97.

Julesz, B., \& Schumer, R. A. (1981). Early visual perception. In M. R. Rosenzweig \& L. W. Porter (Eds.), Annual review of psychology (Vol. 32, pp. 575-627). Palo Alto, CA: Annual Reviews.

KIrBY, N. (1980). Sequential effects in choice reaction times. In A. T. Welford (Ed.), Reaction times (pp. 129-172). New York: Academic Press.

KruSKal, J. B. (1964). Nonmetric multidimensional scaling. Psychometrika, 29, 1-27.

Langley, C. M., Riley, D. A., Bond, A. B., \& Goel, N. (1996). Visual search for natural grains in pigeons (Columba livia): Search images and selective attention. Journal of Experimental Psychology: Animal Behavior Processes, 22, 139-151.

LOGAN, G. D. (1988). Toward an instance theory of automatization. Psychological Review, 95, 492-527.

LUCE, R. D. (1986). Response times: Their role in inferring elementary mental organization. New York: Oxford University Press.

Pietrewicz, A. T., \& Kamil, A. C. (1979). Search image formation in the blue jay (Cyanocitta cristata). Science, 204, 1332-1333.

PODGORNY, P., \& GARNER, W. R. (1979). Reaction time as a measure of inter- and intraobject visual similarity: Letters of the alphabet. Perception \& Psychophysics, 26, 37-52.

PosNer, M. I., \& SNyder, C. R. R. (1975). Facilitation and inhibition in the processing of signals. In P. M. Rabbitt \& S. Dornic (Eds.), $A t$ tention and performance (Vol. 5, pp. 669-682). San Diego, CA: Academic Press.

Prinzmetal, W. (1995). Visual feature integration in a world of objects. Current Directions in Psychological Science, 4, 90-94.

RATCliff, R. (1978). A theory of memory retrieval. Psychological Review, 85, 59-108.

Reid, P. J., \& ShetTleworth, S. J. (1992). Detection of cryptic prey: Search image or search rate? Journal of Experimental Psychology: Animal Behavior Processes, 18, 273-286.

Rosch, E. (1975). The nature of mental codes for color categories. Journal of Experimental Psychology: Human Perception \& Performance, 1, 303-322.

SAS (1982). SAS System [Computer program]. Cary, NC: Author.

SCHILLER, P. H., \& LEE, K. (1991). The role of the primate extrastriate area V4 in vision. Science, 251, 1251-1253.

SCHNEIDER, W., \& Shiffrin, R. W. (1977). Controlled and automatic human information processing: I. Detection, search, and attention. Psychological Review, 84, 1-66.

SHEPARD, R. N. (1962). The analysis of proximities: Multidimensional scaling with an unknown distance function. Psychometrika, 42, 7-67.

SHEPARD, R. N. (1980). Multidimensional scaling, tree-fitting and clustering. Science, 210, 390-398.

SHEPARD, R. N. (1987). Toward a universal law of generalization for psychological science. Science, 237, 1317-1323.

SHIFFrIN, R. W., \& SCHNEIDER, W. (1977). Controlled and automatic human information processing: II. Perceptual learning, automatic attending, and a general theory. Psychological Review, 84, 127-190.

Stebbins, W. C., \& Berkley, M. A. (EDS.) (1990). Comparative perception (Vol. 2). New York: Wiley.

SternberG, S. (1966). High-speed scanning in human memory. Science, 153, 652-654.

Teichner, W. H., \& Krebs, M. J. (1974). Visual search for simple targets. Psychological Bulletin, 31, 15-28.

TiNBERGEN, N. (1960). The natural control of insects in pine woods: I. Factors influencing the intensity of predation by songbirds. $\mathrm{Ar}$ chives Neelandaises de Zoologie, 13, 265-343.

TodRIN, D. C., \& Blough, D. S. (1983). The discrimination of mirrorimage forms by pigeons. Perception \& Psychophysics, 34, 397-402.

Treisman, A. (1986). Properties, parts, and objects. In K. R. Boff, L. Kaufman, \& J. P. Thomas (Eds.), Handbook of perception and human performance (Vol. 2, pp. 1-70). New York: Wiley

Treisman, A., \& Gelade, G. (1980). A feature integration theory of attention. Cognitive Psychology, 12, 97-136. 
Treisman, A., \& Gormican, S. (1988). Feature analysis in early vision. Evidence from search asymmetries. Psychological Review, 95, 15-48.

Treisman, A., Vieira, A., \& Hayes, A. (1992). Automaticity and preattentive processing. American Journal of Psychology, 105, 341-362.

TVersky, A. (1977). Features of similarity. Psychological Review, 84, 327-352.

TVersky, A., \& Gati, I. (1982). Similarity, separability, and the triangle inequality. Psychological Review, 89, 123-154.

VAUGHAN, W., JR., \& GReENE, S. L. (1983). Acquisition of absolute discriminations in pigeons. In M. L. Commons, R. J. Herrnstein, \& A. R. Wagner (Eds.), Quantitative analyses of behavior: Discrimination processes (pp. 231-238). Cambridge, MA: Ballinger.

VREVEN, D., \& Blough, P. M. (1996). Searching for one or many targets: Effects of extended experience on the runs advantage. Manuscript submitted for publication. Providence, RI: Brown University, Department of Psychology.

WASSERMAN, E. A. (1993). Comparative cognition: Beginning the sec- ond century of the study of animal intelligence. Psychological Bulletin, 113, 211-228.

Wasserman, E. A., KirkPatrick-Steger, K., Van Hamme, L. J., \& Biederman, I. (1993). Pigeons are sensitive to the spatial organization of complex visual stimuli. Psychological Science, 4, 336-341.

Williams, B. A. (1988). Reinforcement, choice, and response strength. In R. C. Atkinson, R. J. Herrnstein, G. Lindzey, \& R. D. Luce (Eds.), Stevens' Handbook of experimental psychology (2nd ed., pp. 167244). New York: Wiley.

Wolfe, J. M., CAVe, K. R., \& Franzel, S. L. (1989). Guided search: An alternative to the feature integration model for visual search. Journal of Experimental Psychology: Human Perception \& Performance, 15, 419-433.

ZENTALL, T. R. (ED.) (1993). Animal cognition: A tribute to Donald A. Riley. Hillsdale, NJ: Erlbaum.

(Manuscript received February 15, 1996; revision accepted for publication July 12,1996 .) 